\title{
LOCAL SINGULARITIES SUCH THAT ALL DEFORMATIONS ARE TANGENTIALLY FLAT
}

\author{
BERND HERZOG
}

\begin{abstract}
We give a criterion for a local ring $\left(B_{0}, \mathfrak{n}_{0}\right)$ containing a field to have only tangentially flat deformations. Various examples of such local rings are constructed.
\end{abstract}

Let $\left(B_{0}, \mathfrak{n}_{0}\right)$ be a (Noetherian) local ring. We say that it has only tangentially flat deformations, if every flat local homomorphism of local rings

$$
f:(A, \mathfrak{m}) \rightarrow(B, \mathfrak{n})
$$

such that $B_{0}$ and the (special) fiber $B / \mathrm{m} B$ of $f$ have isomorphic completions $\widehat{B} / \mathrm{m} \widehat{B} \cong \widehat{B}_{0}$, is tangentially flat, i.e., induces a flat homomorphism

$$
\operatorname{gr}(f): \operatorname{gr}(A) \rightarrow \operatorname{gr}(B)
$$

of the associated graded rings with respect to the maximal ideals. Examples of local rings $\left(B_{0}, \mathfrak{n}_{0}\right)$ with this property are the regular local rings and the rings which have completion

$$
\widehat{B}_{0}=L\left[\left[X_{1}, \ldots, X_{N}\right]\right] /\left(X_{1}, \ldots, X_{N}\right)^{d},
$$

where $L$ is a field, $N$ is greater than 1 , and $d$ is an arbitrary positive integer (see [ $\mathrm{He}_{1}$, Folgerung (1.3); $\mathrm{He}_{1}$, Corollary 2]).

In 1981 Christer Lech (Stockholm) stated the problem of whether the local rings $B_{0}$ satisfying (1) are the only zero-dimensional local rings with only tangentially flat deformations. The answer is no, as the following example shows:

$$
B_{0}=L[X, Y, Z] /\left(X^{3}, Y^{3}, Z^{3}, X^{2} Y, Y^{2} Z, Z^{2} X\right)
$$

(see Example (2.7) below). So the next question would be whether it is possible to give a complete list of such singularities, but it turns out there are too many of them.

The purpose of this paper is to give a criterion for local rings $\left(B_{0}, \mathfrak{n}_{0}\right)$ to have only tangentially flat deformations in terms of the normal module $N_{B_{0}}$ of $B_{0}$ (with respect to a formal local embedding into some regular scheme). As

Received by the editors March 6, 1989.

1980 Mathematics Subject Classification (1985 Revision). Primary 13H15, 13D10, 14B07, 14B12. 
an application we give various examples of local singularities $\left(B_{0}, \mathfrak{n}_{0}\right)$ of all dimensions having only tangentially flat deformations.

Note that each such singularity $\left(B_{0}, \mathfrak{n}_{0}\right)$ gives a class of flat local extensions $A \rightarrow B$ of local rings (those with fiber $B_{0}$ ), for which the problem of LechHironaka [L, p. 72; Hi, Problem 10.2$]$ has trivially a positive answer, i.e., the local Hilbert functions $H_{A}^{0}$ and $H_{B}^{0}$ of $A$ and $B$ respectively satisfy

$$
H_{A}^{d}(n) \leq H_{B}^{0}(n) \text { for } n \in \mathbf{N} \text { and } d:=\operatorname{dim} B_{0} .
$$

Throughout the paper we use the standard assumptions and notation of commutative algebra as in [Ma, Bo] (unless stated otherwise). Local rings will always be Noetherian.

\section{SOME CRITERIA FOR TANGENTIAL FLATNESS}

(1.1) Definition. Let $G=\bigoplus_{n \geq 0} G(n)$ be a (commutative) graded algebra of finite type over the Artin local ring $G(0)$ of elements of degree zero. Then the Hilbert series $H_{G}$ of $G$ is the formal power series $H_{G}=\sum_{n \in \mathbf{Z}} H_{G}(n) T^{n}$ with integer coefficients

$$
H_{G}(n):= \begin{cases}\text { length }_{G(0)} G(n) & \text { for } n \geq 0, \\ 0 & \text { otherwise. }\end{cases}
$$

The $i$ th sum transform of the Hilbert series of $G$ is defined to be

$$
H_{G}^{i}:=\sum_{n \in \mathbf{Z}} H_{G}^{i}(n) T^{n}:=(1-T)^{-i} H_{G} .
$$

Given any two formal power series $H=\sum H(n) T^{n}$ and $H^{\prime}=\sum H^{\prime}(n) T^{n}$ with integer coefficients, the inequality $H \leq H^{\prime}$ is to be understood in the sense of total order, i.e., $H(n) \leq H^{\prime}(n)$ for all $n$.

Let $(A, \mathfrak{m})$ be a local ring. Then the Hilbert series of $A$ and its sum transforms are defined to be

$$
H_{A}:=H_{\operatorname{gr}(A)} \quad \text { and } \quad H_{A}^{i}:=H_{\mathrm{gr}(A)}^{i}
$$

respectively. Here $\operatorname{gr}(A)$ denotes the associated graded ring with respect to the powers of the maximal ideal $\mathfrak{m}$ of $A$.

The essential part of the proof of the following theorem can be found in $\left[\mathrm{He}_{2}\right]$.

(1.2) Theorem. (i) Let $f:(A, \mathfrak{m}, K) \rightarrow(B, \mathfrak{n}, L)$ be a local homomorphism of local rings with special fiber $B_{0}:=B / \mathrm{m} B$. Then

$$
H_{B}^{1} \leq H_{A}^{1} H_{B_{0}}^{0} .
$$

(ii) Equality holds in (i) if and only if one of the following equivalent conditions is satisfied.

(a) $H_{\mathrm{gr}(B)}^{1}=H_{\mathrm{gr}(A)}^{1} \cdot H_{\mathrm{gr}(B) \otimes_{\mathrm{gr}(A)} K}^{0}$. 
(b) The homomorphism $f$ is flat and one has the identities $\mathfrak{m}^{i} B \cap \mathfrak{n}^{j}=$ $\mathfrak{m}^{i} \mathfrak{n}^{j-i}$ for $j \geq i$.

(c) The homomorphism $f$ is flat and the canonical surjections

$$
\operatorname{gr}(B) / \mathrm{gr}^{\geq i}(A) \operatorname{gr}(B) \rightarrow \operatorname{gr}\left(B / \mathrm{m}^{i} B\right)
$$

are bijective for all $i$. Here $\mathrm{gr}^{\geq i}(A)$ denotes the ith power of the irrelevant maximal ideal $\operatorname{gr}^{+}(A)=\bigoplus_{k \geq 1} \mathfrak{m}^{k} / \mathfrak{m}^{k+1}$ of $\operatorname{gr}(A)$.

(d) There is a graded L-module section $s$ of the canonical homomorphism $\operatorname{gr}(B) \rightarrow \operatorname{gr}\left(B_{0}\right)$ such that the induced homomorphism of graded $\operatorname{gr}(A) \otimes_{K}$ $L$-modules

$$
\operatorname{gr}(A) \otimes_{K} \operatorname{gr}\left(B_{0}\right) \rightarrow \operatorname{gr}(B)
$$

is injective (in which case it is bijective for any such section $s$ ).

(e) The homomorphism $\operatorname{gr}(f): \operatorname{gr}(A) \rightarrow \operatorname{gr}(B)$ induced by $f$ is flat, i.e., $f$ is tangentially flat.

Conditions (b) and (c) are satisfied, provided one of them is satisfied for $i=1$. Proof. Assertion (i) is just Theorem 1 of $\left[\mathrm{He}_{2}\right]$. From Theorem 2 of $\left[\mathrm{He}_{2}\right]$ we know that equality in (i) is equivalent to any of the conditions (b), (d), and (e). Condition (c) is simply a reformulation of $(b)$. To see this, observe that

$$
\begin{aligned}
\operatorname{gr}\left(B / \mathfrak{m}^{i} B\right) & =\bigoplus_{j \geq 0}\left(\mathfrak{n}^{j}+\mathfrak{m}^{i} B\right) /\left(\mathfrak{n}^{j+1}+\mathfrak{m}^{i} B\right) \\
& =\bigoplus_{j \geq 0} \mathfrak{n}^{j} /\left(\mathfrak{n}^{j+1}+\mathfrak{m}^{i} B \cap \mathfrak{n}^{j}\right)
\end{aligned}
$$

and

$$
\operatorname{gr}(B) / \operatorname{gr}^{\geq i}(A) \operatorname{gr}(B)=\bigoplus_{j \geq 0} \mathfrak{n}^{j} /\left(\mathfrak{n}^{j+1}+\mathfrak{m}^{i} \mathfrak{n}^{j-i}\right) .
$$

So the canonical surjections of (c) are bijective if and only if

$$
\mathfrak{m}^{i} B \cap \mathfrak{n}^{j}=\mathfrak{m}^{i} \mathfrak{n}^{j-i}+\mathfrak{m}^{i} B \cap \mathfrak{n}^{j+1} .
$$

An easy induction shows that this is equivalent to the identities of (b) (use the lemmas of Artin-Rees and Nakayama). We have proved (b) $\Leftrightarrow(\mathrm{c}) \Leftrightarrow(\mathrm{d}) \Leftrightarrow(\mathrm{e})$.

Condition (a) is a consequence of (d) and (c). For, condition (d) implies

$$
\begin{aligned}
H_{\mathrm{gr} B}^{0}(n) & =\text { length } \bigoplus_{i+j=n} \operatorname{gr}^{i}(A) \otimes_{K} \operatorname{gr}^{j}\left(B_{0}\right) \\
& =\sum_{i+j=n} H_{\operatorname{gr}(A)}^{0}(i) H_{\operatorname{gr}\left(B_{0}\right)}^{0}(j),
\end{aligned}
$$

i.e., $H_{\mathrm{gr}(B)}^{0}=H_{\mathrm{gr}(A)}^{0} H_{\mathrm{gr}\left(B_{0}\right)}^{0}$ and therefore

$$
H_{\mathrm{gr}(B)}^{1}=H_{\mathrm{gr}(A)}^{1} H_{\mathrm{gr}\left(B_{0}\right)}^{0} .
$$

This is equivalent to (a), since by condition (c)

$$
\operatorname{gr}\left(B_{0}\right)=\operatorname{gr}(B / \mathfrak{m} B) \cong \operatorname{gr}(B) / \operatorname{gr}^{+}(A) \operatorname{gr}(B) \cong \operatorname{gr}(B) \otimes_{\operatorname{gr}(A)} K .
$$


Conversely, from $\left[\mathrm{He}_{1}\right.$, Satz (1.1)] we know that (a) implies (e).

Now all we have to prove is the remark at the end of the theorem that conditions (b) and (c) are satisfied if one of them is satisfied for $i=1$. This is done in $\left[\mathrm{He}_{3}\right]$. But there is a much more elementary proof due to the late Professor Lech; we give a reformulation of this here. All we have to show is the following lemma.

(1.3) Lemma (Lech). Let $f:(A, \mathfrak{m}) \rightarrow(B, \mathfrak{n})$ be a flat local homomorphism such that $\mathfrak{m} B \cap \mathfrak{n}^{j}=\mathfrak{m n}^{j-1}$ for all $j$. Then

$$
\mathfrak{m}^{i} B \cap \mathfrak{n}^{j}=\mathfrak{m}^{i} \mathfrak{n}^{j-i} \text { for } j \geq i .
$$

Proof. The case $i=1$ is trivial, so assume $i>1$. By the induction hypothesis,

$$
\mathfrak{m}^{i} B \cap \mathfrak{n}^{j}=\mathfrak{m}^{i} B \cap \mathfrak{m}^{i-1} B \cap \mathfrak{n}^{j}=\mathfrak{m}^{i} B \cap\left(\mathfrak{m}^{i-1} \mathfrak{n}^{j-i+1}\right) .
$$

Fix some minimal set of generators $x_{1}, \ldots, x_{N}$ of the ideal $\mathfrak{m}^{i-1}$. Then any element $y$ of the left-hand side of (2) can be written as

$$
y=\sum a_{k} x_{k}=\sum b_{k} x_{k}
$$

with $a_{k} \in \mathfrak{m} B$ and $b_{k} \in \mathfrak{n}^{j-i+1}$. In particular, $\sum\left(a_{k}-b_{k}\right) x_{k}=0$. Since $f: A \rightarrow B$ is flat and the $x_{k}$ 's form a minimal generating set of $\mathrm{m}^{i-1}$, this implies

$$
\begin{aligned}
a_{k}-b_{k} & \in\left(x_{1}, \ldots \hat{x}_{k} \ldots, x_{N}\right) B: x_{k} \\
& =\left[\left(x_{1}, \ldots \hat{x}_{k} \ldots, x_{N}\right) A: x_{k}\right] B \subseteq \mathfrak{m} B .
\end{aligned}
$$

Here a hat " $\leadsto$ " on $x_{k}$ means that this element is to be omitted. We see that $b_{k}$ is in $\mathfrak{m} B$, hence in $\mathfrak{m} B \cap \mathfrak{n}^{j-i+1}$. Therefore

$$
y=\sum b_{k} x_{k} \in \mathfrak{m} \mathfrak{n}^{j-i} \cdot \mathfrak{m}^{i-1}=\mathfrak{m}^{i} \mathfrak{n}^{j-i} .
$$

We have proved that the left-hand side of (2) is contained in the right-hand side. The converse inclusion is trivial. Q.E.D.

(1.4) Remarks. (i) Let the local homomorphism of local rings

$$
f:(A, \mathfrak{m}) \rightarrow(B, \mathfrak{n})
$$

be tangentially flat. Then so is $f \otimes_{A} A / I$ for every ideal $I \subseteq \mathfrak{m}$.

(ii) Let $f:(A, \mathfrak{m}) \rightarrow(B, \mathfrak{n})$ be a local homomorphism of local rings and let $I_{1} \supseteq I_{2} \supseteq I_{3} \supseteq \cdots$ be a descending chain of ideals of $A$ such that given any power $\mathrm{m}^{k}$ of the maximal ideal of $A$, there is some $I_{j}$ contained in $\mathrm{m}^{k}$. Then the following conditions are equivalent.

(a) $f$ is tangentially flat.

(b) $f \otimes_{A} A / I_{j}$ is tangentially flat for every $j$. 
(iii) Let $f:(A, \mathfrak{m}) \rightarrow(B, \mathfrak{n})$ be a local homomorphism of Artin local rings. Then the following conditions are equivalent.

(a) $f$ is tangentially flat.

(b) $H_{B}^{0} \leq H_{A}^{0} H_{B_{0}}^{0}$ and $f$ is flat.

(c) $H_{B}^{0} \geq H_{A}^{0} H_{B_{0}}^{0}$.

Here as usual $B_{0}$ is the special fiber $B / \mathrm{m} B$ of $f$.

Proof. For assertion (i) see $\left[\mathrm{He}_{2}\right.$, Remark 1]. To prove assertion (ii) assume condition (b) is satisfied. Then

$$
H_{B / I_{j} B}^{1}(k)=\sum_{u+v=k} H_{A / I_{j}}^{1}(u) H_{B_{0}}^{0}(v)
$$

for every $k$ and every $j$. For fixed $k$ and $j$ large enough $I_{j}$ is contained in $\mathfrak{m}^{k+1}$, hence

$$
H_{A / I_{j}}^{1}(u)=H_{A}^{1}(u) \quad \text { for } u=0,1, \ldots, k
$$

and

$$
H_{B / I_{j} B}^{1}(k)=H_{B}^{1}(k) .
$$

Therefore $H_{B}^{1}=H_{A}^{1} H_{B_{0}}^{0}$, i.e., $f: A \rightarrow B$ is tangentially flat. We have proved that condition (b) of (ii) implies condition (a). The converse implication follows from (i).

To prove (iii), note that condition (a) implies (b) and (c). Conversely, assume that (b) or (c) is true. All we have to show is that then equality holds in (b) and (c) respectively. For this it is sufficient to take the sum over all coefficients of the series on both sides of (b) and (c) respectively to obtain an identity, i.e., we have only to show

$$
\sum_{k=0}^{\infty} H_{B}^{0}(k)=\sum_{k=0}^{\infty} \sum_{u+v=k} H_{A}^{0}(u) H_{B_{0}}^{0}(v) .
$$

Note that since $A$ and $B$ are Artin local rings, both sides are finite. The lefthand side of (3) is equal to length ${ }_{B}(B)$ and the right-hand side equals

$$
\sum_{u=0}^{\infty} H_{A}^{0}(u) \sum_{v=0}^{\infty} H_{B_{0}}^{0}(v)=\text { length }_{A}(A) \cdot \text { length }_{B}(B / \mathrm{m} B) .
$$

Therefore (3) follows from the fact that $B$ is a flat module over $A$ in case (b) and is trivial in case (c). Q.E.D.

(1.5) Theorem. A local homomorphism $f:(A, \mathfrak{m}, K) \rightarrow(B, \mathfrak{n}, L)$ is tangentially flat if and only if the following conditions are satisfied.

(i) $f$ is flat.

(ii) $\operatorname{ord}_{B}(a b)=\operatorname{ord}_{A}(a)+\operatorname{ord}_{B}(b)$ for every $a \in A$ and for every $b \in B$ such that $\operatorname{ord}_{B}(b)=\operatorname{ord}_{B_{0}}\left(b_{0}\right)$. Here $B_{0}:=B / \mathfrak{m} B$ is the special fiber of $f, b_{0}$ is 
the residue class of $b$ in $B_{0}$, and ord is the order with respect to the maximal ideal in the corresponding local ring.

(iii) Condition (ii) is preserved if $f$ is replaced by $f \otimes_{A} A / I$ for every proper ideal I of $A$.

Proof. Suppose $f$ is tangentially flat. Then $f$ is flat by Theorem (1.2). Since tangential flatness is preserved when passing from $f$ to $f \otimes_{A} A / I$ (see Remark (1.4)(i)), it is sufficient to prove that condition (ii) is satisfied. In proving this, we may assume $a$ and $b$ are both nonzero. Then the initial form $\operatorname{in}(b)$ of $b$ in $\operatorname{gr}(B)$ represents a nonzero element of the $K$ vector space $\operatorname{gr}(B) / \operatorname{gr}^{+}(A) \operatorname{gr}(B) \cong \operatorname{gr}\left(B_{0}\right)$, hence a member of a family of homogeneous and linearly independent generators of this vector space. Lifting these generators to homogeneous elements of $\operatorname{gr}(B)$, one obtains a free generating set of $\operatorname{gr}(B)$ over $\operatorname{gr}(A)$, and lifting these to $B$, one sees that there is a family $\left(b_{i}\right)_{i \in I}$ of elements $b_{i} \in B$ such that

1. $b$ is a member of $\left(b_{i}\right)_{i \in I}$.

2. $B \subseteq \sum_{i \in I} A b_{i}+\mathfrak{n}^{k}$ for every $k$.

3. $\sum_{i \in I} a_{i} b_{i} \in \mathfrak{n}^{k}, a_{i} \in A$, implies $a_{i} \in \mathfrak{m}^{k-d(i)}$ for every $i \in I$, where $d(i):=\operatorname{ord}_{B}\left(b_{i}\right)$.

Let $\operatorname{ord}_{A}(a)=r, \operatorname{ord}_{B}(b)=s$, and $\operatorname{ord}_{B}(a b)=t$. Then (1) and (3) imply $a \in m^{t-s}$, i.e., $r+s \geq t$. Since the converse inequality is trivial, this is the claim of (ii).

Now suppose conditions (i)-(iii) are satisfied. Since tangential flatness of $f$ is equivalent to tangential flatness of $f \otimes_{A} A / \mathrm{m}^{k}$ for every $k$, we may assume $A$ is an Artin local ring. The further proof uses the proposition of $\left[\mathrm{He}_{3}\right]$. From this proposition we see that there are families $\left(b_{j i}\right)_{i \in I_{j}}(j=0,1,2, \ldots)$ of elements $b_{i j} \in B$ such that

4. $\operatorname{ord}_{B}\left(b_{j i}\right)=\operatorname{ord}_{B_{0}}\left(b_{j i} \bmod \mathfrak{m} B\right)=j$.

5. The homomorphism of $A$-modules

$$
\prod_{j=0}^{\infty} \bigoplus_{i \in I_{j}} A \rightarrow \widehat{B}, \quad\left(a_{j i}\right) \mapsto \sum a_{j i} b_{j i}
$$

is well defined and bijective (since $f$ is flat).

6. $f$ is tangentially flat if and only if $\sum a_{j i} b_{j i} \in \mathfrak{n}^{k} \widehat{B}$ implies $a_{j i} \in \mathfrak{m}^{k-j}$ for all $i \in I_{j}$ and all $j$.

7. For any given $j$ the $b_{j i}$ 's represent $K$-linearly independent elements of $\mathfrak{n}^{j} B_{0} / \mathfrak{n}^{j+1} B_{0}$.

Assume that $f$ is not tangentially flat. Then by property 6 there is an element

$$
b=\sum a_{j i} b_{j i} \in \widehat{B}
$$

such that $\operatorname{ord}_{A}\left(a_{j i}\right)+\operatorname{ord}_{B}\left(b_{j i}\right)<\operatorname{ord}_{\widehat{B}}(b)$ for at least one pair $(j, i)$. Define

$$
u:=\min \left(\operatorname{ord}_{A}\left(a_{j i}\right)+\operatorname{ord}_{B}\left(b_{j i}\right)\right)
$$


and omit in the sum (4) all terms but those satisfying

$$
\operatorname{ord}_{A}\left(a_{j i}\right)+\operatorname{ord}_{B}\left(b_{j i}\right)=u \text {. }
$$

Then the sum (4) becomes finite, since for any given $j$ there are only finitely many nonzero coefficients $a_{j i}$. In particular, $b$ is in $B$. Let

$$
N:=\#\left\{(j, i) \mid a_{j i} \neq 0\right\}
$$

be the number of nonzero members in the sum (4) and let $r:=\min _{A} \operatorname{ord}_{A}\left(a_{j i}\right)$. Replacing $f$ by $f \otimes_{A} A / \mathfrak{m}^{r+1}$, the orders of the elements $b_{j i}$ do not increase by property 4 , so equality (5) remains true. Therefore we may assume

$$
r=\operatorname{ord}_{A}\left(a_{j i}\right) \text { for every nonzero } a_{j i} \text {. }
$$

From equations (5) and (6) we see that $a_{j i} \neq 0$ only if $j=u-r$.

In case there are two pairs $(j, i)$ and $\left(j^{\prime}, i^{\prime}\right)$ such that $a_{j^{\prime} i^{\prime}} \notin a_{j i} A$, the homomorphism $f$ can be replaced by $f \otimes_{A} A / a_{j i} A$ with the effect that one of these coefficients becomes zero, i.e., $N$ decreases. This can be done only a finite number of times. So we may assume that any two nonzero coefficients $a_{j i}$ are proportional, i.e., $a_{j i}=e_{j i} a$ with $a \in A$ fixed and $e_{j i}$ a unit for some pairs $(j, i)$ satisfying $j=u-r$ and zero otherwise. Then

$$
b=a b^{\prime}, \quad b^{\prime}:=\sum_{i} e_{u-r, i} b_{u-r, i} .
$$

From property 7 we see that $b^{\prime}$ has order

$$
\operatorname{ord}_{B}\left(b^{\prime}\right)=\operatorname{ord}_{B_{0}}\left(b^{\prime} \bmod \mathfrak{m} B\right)=u-r .
$$

By construction, $\operatorname{ord}_{A}(a)=r$. Therefore

$$
\operatorname{ord}_{B}\left(a b^{\prime}\right)=\operatorname{ord}_{B}(b)>u=r+(u-r)=\operatorname{ord}_{A}(a)+\operatorname{ord}_{B}\left(b^{\prime}\right) .
$$

This contradicts our assumptions (ii) and (iii). Hence $f$ is tangentially flat. Q.E.D.

(1.6) Proposition. Let $f:(A, \mathfrak{m}, K) \rightarrow(B, \mathfrak{n}, L)$ be a flat local homomorphism of complete local rings. Then there is a commutative diagram of local homomorphisms

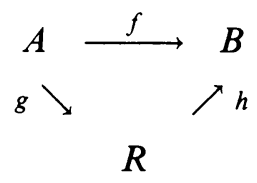

such that $g$ is tangentially flat and $h$ is surjective.

Proof. From the theory of Cohen rings (see [GD, EGA IV $\left.{ }_{1}, \S 19\right]$ ) it is easily seen that there is a commutative diagram of local homomorphisms

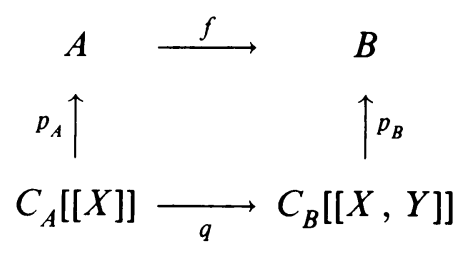


with $X$ and $Y$ finite sets of indeterminates, $C_{A}$ and $C_{B}$ Cohen rings, $p_{A}$ and $p_{B}$ surjections, and $q$ a homomorphism inducing the identity on $X$. So $A$ and $B$ can be identified with factor rings of $C_{A}[[X]]$ and $C_{B}[[X, Y]]$ respectively. This gives a commutative diagram

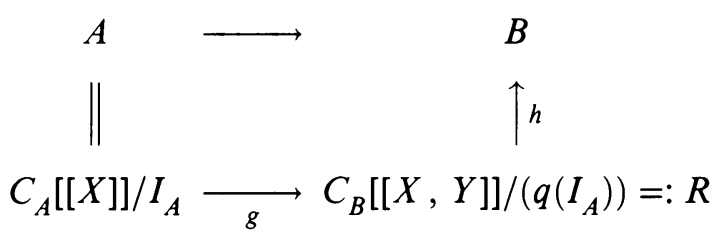

with $g$ and $h$ induced by $q$ and $p_{B}$ respectively and $I_{A}$ the kernel of $p_{A}$. The homomorphism $h$ is obviously surjective. All we have left to prove is the tangential flatness of $g$. Note that $g$ is flat (since $q$ is flat). So it is sufficient to show that the special fiber of $g$ is a regular local ring (see [He ${ }_{1}$, Folgerung (1.3)]). But the fiber of $g$ coincides with the fiber of $q$, which is obviously regular. Q.E.D.

(1.7) Terminology. A standard base of an ideal $I$ in a local ring $(R, \mathfrak{M})$ is defined to be a sequence $r=\left(r_{1}, \ldots, r_{n}\right)$ of elements of $I$ such that the corresponding sequence

$$
\operatorname{in}(r):=\left(\operatorname{in}\left(r_{1}\right), \ldots, \text { in }\left(r_{n}\right)\right)
$$

of initial forms in $\operatorname{gr}(R)$ generate the initial ideal

$$
\operatorname{gr}(I, R):=\bigoplus_{k \geq 0} I \cap \mathfrak{M}^{k}+\mathfrak{M}^{k+1} / \mathfrak{M}^{k+1} .
$$

For any two $n$-tuples $r, s \in R^{n}$ we write

$$
\langle r, s\rangle:=\sum_{i=1}^{n} r_{i} s_{i}
$$

and more generally

$$
\langle A, s\rangle:=\left(\left\langle r^{1}, s\right\rangle, \ldots,\left\langle r^{n}, s\right\rangle\right)
$$

for any $n \times n$-matrix $A$ over $R$ with rows $r^{1}, \ldots, r^{n}$. Further let

$$
\operatorname{ord}(r):=\left(\operatorname{ord}_{R}\left(r_{1}\right), \ldots, \operatorname{ord}_{R}\left(r_{n}\right)\right)
$$

for $r=\left(r_{1}, \ldots, r_{n}\right) \in R^{n}$. If $r \in R$, we write $(r):=(r, \ldots, r)$ for the $n$-tuple with all coordinates equal to $r$. It will be clear from the context which $n$ we have in mind. In case the coordinates of $r$ and $s$ are integers the inequality $r=\left(r_{1}, \ldots, r_{n}\right) \leq s=\left(s_{1}, \ldots, s_{n}\right)$ means that $r_{i} \leq s_{i}$ for every $i$.

(1.8) Theorem. Let the local homomorphism $f:(A, \mathfrak{M}, k) \rightarrow(R, \mathfrak{M}, L)$ be tangentially flat and let the ideal $I \subseteq \mathfrak{m}$ be such that $B:=R / I$ is flat over $A$. Then the following are equivalent. 
(i) $B$ is tangentially flat over $A$.

(ii) There is a standard base $r_{0}$ of the ideal $I_{0}:=I R_{0}$ of $R_{0}:=R / \mathrm{m} R$ that can be lifted to an $n$-tuple $r$ of elements from $I$ such that $\operatorname{ord}(r)=$ $\operatorname{ord}\left(r_{0}\right)$ (in which case any such lift $r$ of any standard base $r_{0}$ is automatically a standard base of $I$ ).

(iii) Every element $x_{0} \in I_{0}$ can be lifted to an element $x \in I$ such that $\operatorname{ord}_{R}(x)=\operatorname{ord}_{R_{0}}\left(x_{0}\right)$.

Proof. (i) $\Rightarrow$ (iii) Consider the commutative diagram with exact rows

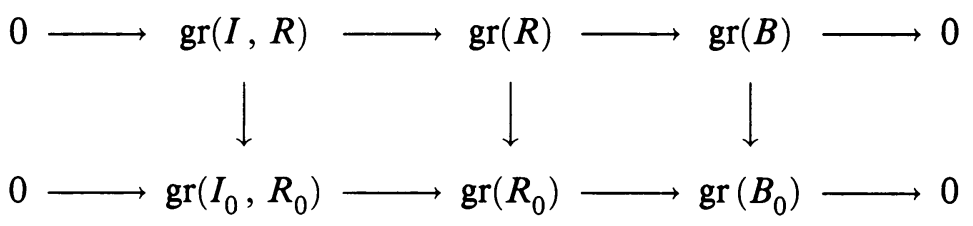

where $B_{0}:=B / \mathrm{m} B$ is the special fiber of $A \rightarrow B$ and the vertical homomorphisms are the canonical ones. Note that the latter are surjective. This is obvious for those on the right and in the middle. For the left-hand side homomorphism it follows from the fact that

$$
\begin{aligned}
\operatorname{gr}\left(R_{0}\right) / \operatorname{gr}(I, R) \operatorname{gr}\left(R_{0}\right) & \cong \operatorname{gr}(R) / \operatorname{gr}(I, R)+\left(\operatorname{gr}^{+}(A)\right) \\
& \cong \operatorname{gr}(B) /\left(\operatorname{gr}^{+}(A)\right) \cong \operatorname{gr}\left(B_{0}\right) .
\end{aligned}
$$

Here the first isomorphy comes from the fact that $R$ is tangentially flat over $A$ (see Theorem $(1.2)(\mathrm{c}))$ and the third is a consequence of the tangential flatness of $B$ over $A$.

The surjectivity of the left-hand side vertical homomorphism may be interpreted as follows: for every positive integer $k$ one has the inclusion

$$
(I+\mathfrak{m} R) \cap \mathfrak{M}^{k} \subseteq(I+\mathfrak{m} R) \cap \mathfrak{M}^{k+1}+I \cap \mathfrak{M}^{k}+\mathfrak{m} R .
$$

The Artin-Rees lemma implies

$$
(I+\mathfrak{m} R) \cap \mathfrak{M}^{k} \subseteq I \cap \mathfrak{M}^{k}+\mathfrak{m} R,
$$

i.e., every element from $I_{0}$ of order $k$ can be lifted to an element of $I$ with the same order.

(iii) $\Rightarrow$ (ii) This is trivial, except for the assertion added in parentheses, which will be proved later.

(ii) $\Rightarrow$ (i) It is sufficient to show that the canonical surjection $\operatorname{gr}(B) /\left(\mathrm{gr}^{+}(A)\right)$ $\rightarrow \operatorname{gr}\left(B_{0}\right)$ is bijective. The following composition of canonical maps is an inverse:

$$
\begin{aligned}
\operatorname{gr}\left(B_{0}\right) & \stackrel{\sim}{\rightarrow} \operatorname{gr}\left(R_{0}\right) / \operatorname{gr}\left(I_{0}, R_{0}\right) \\
& =\operatorname{gr}\left(R_{0}\right) / \operatorname{in}(r) \operatorname{gr}\left(R_{0}\right) \\
& \stackrel{\sim}{\rightarrow} \operatorname{gr}(R) / \operatorname{in}(r) \operatorname{gr}(R)+\left(\mathrm{gr}^{+}(A)\right) \\
& \rightarrow \operatorname{gr}(R) / \operatorname{gr}(I, R)+\left(\mathrm{gr}^{+}(A)\right) \\
& \stackrel{\sim}{\rightarrow} \operatorname{gr}(B) /\left(\mathrm{gr}^{+}(A)\right) .
\end{aligned}
$$


Note that the isomorphism in the third row exists, since $R$ is tangentially flat over $A$. We have proved tangential flatness of $B$ over $A$.

Since the inverse mapping given above is bijective, the following identity holds in $\operatorname{gr}(R)$ :

$$
\operatorname{in}(r) \operatorname{gr}(R)+\left(\operatorname{gr}^{+}(A)\right)=\operatorname{gr}(I, R)+\left(\operatorname{gr}^{+}(A)\right) .
$$

Therefore,

$$
\operatorname{gr}(I, R)=\operatorname{in}(r) \operatorname{gr}(R)+\operatorname{gr}(I, R) \cap\left(\operatorname{gr}^{+}(A)\right) .
$$

Since $\operatorname{gr}(R) / \operatorname{gr}(I, R) \cong \operatorname{gr}(B)$ is flat over $\operatorname{gr}(A)$, the second term on the right can be written $\operatorname{gr}^{+}(A) \cdot \operatorname{gr}(I, R)$, so that

$$
\operatorname{gr}(I, R)=\operatorname{in}(r) \operatorname{gr}(R),
$$

i.e., $r$ is a standard base of $I$. Q.E.D.

\section{TANGENTIAL FLATNESS AND THE NORMAL MODULE}

In this section we give the announced characterization of local singularities with only tangentially flat deformations.

(2.1) Definition. Let $R$ be a ring (commutative with unity) and $I$ an ideal of $R$. Then the module

$$
N_{i}:=\operatorname{Hom}_{R}(I, R / I) \quad\left(=\operatorname{Hom}_{R / I}\left(I / I^{2}, R / I\right)\right)
$$

will be called the normal module of $I$. In case $R$ is equipped with some descending filtration

$$
F_{R}: \cdots \supseteq F_{R}^{d} \supseteq F_{R}^{d+1} \supseteq \cdots
$$

by $R$-submodules (i.e., ideals), then the same is true for $N_{I}$, where the $d$ th submodule of the filtration is

$$
F_{N_{I}}^{d}:=\left\{f \in N_{I} \mid f\left(I \cap F_{R}^{k}\right) \subseteq F_{R}^{k+d}+I / I \text { for every } k\right\} .
$$

The associated graded module will be denoted

$$
\operatorname{gr}\left(N_{I}\right):=\bigoplus_{d \in \mathbf{Z}} F_{N_{I}}^{d} / F_{N_{I}}^{d+1}
$$

In case $(R, \mathfrak{M})$ is a local ring, we will always assume that $F_{R}$ is the canonical filtration defined by the powers of the maximal ideal, i.e.,

$$
F_{R}^{d}= \begin{cases}\mathfrak{M}^{d} & \text { for } d>0, \\ R & \text { otherwise }\end{cases}
$$

Now assume $R=\bigoplus_{d=0}^{\infty} R(d)$ is a graded ring and $I=\bigoplus_{d=0}^{\infty} I(d)$ is a homogeneous ideal with $R(d)$ and $I(d)$ the submodules over $R(0)$ of homogeneous elements of degree $d$. Then, if $R$ is Noetherian,

$$
N_{I}=\bigoplus_{d \in \mathbf{Z}} N_{I}(d)
$$


is a graded $R$-module with

$$
N_{I}(d):=\left\{f \in N_{I} \mid f(I(k)) \subseteq R(k+d) / I(k+d) \text { for every } k\right\}
$$

the submodule of homogeneous elements of degree $d$. For any graded $R$ module $N=\bigoplus_{d \in \mathbf{Z}} N(d)$ and any integer $n$ let $N(<n):=\bigoplus_{d<n} N(d)$.

The proposition below gives conditions for tangential flatness of the small prolongations of a given tangentially flat deformation. It represents the crucial step in the proof of our criterion.

(2.2) Proposition. Let $f:(A, \mathfrak{m}, K) \rightarrow(R, \mathfrak{M}, L)$ be a tangentially flat local homomorphism of local rings, $t \in A$ an element in the socle of $A$ (i.e., such that $\mathfrak{m} t=(0))$, and $I$ an ideal of $R$ such that $B:=R / I$ is flat over $A$. Suppose $\bar{B}:=$ $B / t B$ is tangentially flat over $\bar{A}:=A / t A$ and consider the following conditions.

(i) $N_{\mathrm{gr}\left(I_{0}, R_{0}\right)}\left(<-\operatorname{ord}_{A}(t)\right)=0$. Here $R_{0}:=R / \mathrm{m} R$ and $I_{0}:=I R_{0}$.

(ii) For every ideal $I^{\prime}$ of $R$ such that $I+t R=I^{\prime}+t R$ and such that $B^{\prime}:=R / I^{\prime}$ is flat over $A$, the ring $B^{\prime}$ is tangentially flat over $A$.

(iii) $\operatorname{gr}\left(N_{I_{0}}\right)\left(<-\operatorname{ord}_{A}(t)\right)=0$.

Then the following implications (i) $\Rightarrow$ (ii) $\Rightarrow$ (iii) hold.

Proof. (i) $\Rightarrow$ (ii) We have to show that $B^{\prime}=R / I^{\prime}$ is tangentially flat over $A$. For this it is sufficient to prove that $I^{\prime}$ satisfies condition (ii) of Theorem (1.8). Define $\bar{R}:=R / t R$ and $\bar{I}:=I \bar{R}=I^{\prime} \bar{R}$, and let $r_{0} \in R_{0}^{n}$ be a standard base of $I_{0}$. Since $\bar{B}$ is tangentially flat over $\bar{A}, r_{0}$ can be lifted to a standard base $\bar{r} \in \bar{R}^{n}$ of $\bar{I}$ satisfying $\operatorname{ord}(\bar{r})=\operatorname{ord}\left(r_{0}\right)$. In particular the coordinates of $\bar{r}$ generate $\bar{I}$. Since $B^{\prime}$ is flat over $A$, there is a lift $r^{\prime} \in R^{n}$ of $\bar{r}$ such that the coordinates of $r^{\prime}$ generate $I^{\prime}$. Write

$$
r^{\prime}=r+t s_{0}, \quad r \in R^{n}, s_{0} \in R_{0}^{n},
$$

such that

$$
\operatorname{ord}(r)=\operatorname{ord}(\bar{r})=\operatorname{ord}\left(r_{0}\right) .
$$

Note that $t s_{0}$ is a well-defined element of $R^{n}$, since $t$ annihilates $\mathrm{m} R$.

Let $e:=\operatorname{ord}_{A}(t)$ and assume the lift $r^{\prime}$ and its decomposition $r^{\prime}=r+t s_{0}$ are such that

$$
\operatorname{ord}\left(s_{0}\right) \geq \operatorname{ord}\left(r_{0}\right)-(e) .
$$

Then $\operatorname{ord}\left(r^{\prime}\right)=\operatorname{ord}\left(r_{0}\right)$, i.e., $I^{\prime}$ satisfies condition (ii) of Theorem (1.8) and $B^{\prime}$ is tangentially flat over $A$.

So assume that (7) is wrong and choose the minimal integer $m$ such that

$$
\operatorname{ord}\left(s_{0}\right) \geq \operatorname{ord}\left(r_{0}\right)-(m) \text {. }
$$

Then $m>e$. It will be sufficient to prove that in this situation the decomposition $r^{\prime}=r+t s_{0}$ can be replaced by another one with smaller $m$. We shall use 
the following notation:

$$
\begin{gathered}
\operatorname{ord}\left(r_{0}\right)=:(d(1), \ldots, d(n)), \\
\mathfrak{M}_{0}:=\mathfrak{M}_{0}, \\
S_{0}:=s_{0} \bmod \left(\mathfrak{M}_{0}^{d(1)-m+1} \oplus \cdots \oplus \mathfrak{M}_{0}^{d(n)-m+1}\right) \in \operatorname{gr}\left(R_{0}\right)^{n} .
\end{gathered}
$$

As we shall prove below the $n$-tuple $S_{0}$ defines an element

$$
\left\langle H_{0}, \operatorname{in}\left(r_{0}\right)\right\rangle \mapsto \text { residue class of }\left\langle H_{0}, S_{0}\right\rangle
$$

of the normal module

$$
N_{\operatorname{gr}\left(I_{0}, R_{0}\right)}=\operatorname{Hom}_{\operatorname{gr}\left(R_{0}\right)}\left(\operatorname{gr}\left(I_{0}, R_{0}\right), \operatorname{gr}\left(R_{0}\right) / \operatorname{gr}\left(I_{0}, R_{0}\right)\right) .
$$

Note that, since $r_{0}$ is a standard base of $I_{0}$, the elements of $\operatorname{gr}\left(I_{0}, R_{0}\right)$ can be written $\left\langle H_{0}\right.$, in $\left.\left(r_{0}\right)\right\rangle$ with $H_{0} \in \operatorname{gr}\left(R_{0}\right)^{n}$.

The element (8), if it exists, is homogeneous, since $\operatorname{deg} S_{0}-\operatorname{deg} \operatorname{in}\left(r_{0}\right)=$ $(-m, \ldots,-m)$. Its degree is $-m$, hence less than $-e=-\operatorname{ord}_{A}(t)$. From our hypothesis (i) we see that the element (8) must be zero, i.e., the coordinates of $S_{0}$ must be in $\operatorname{gr}\left(I_{0}, R_{0}\right)$. Since $r_{0}$ is a standard base of $I_{0}$, there is a matrix $A$ with elements taken from $R_{0}$ such that the $n$-tuple $s_{0}^{\prime}:=s_{0}-\left\langle A, r_{0}\right\rangle$ satisfies

$$
\operatorname{ord}\left(s_{0}^{\prime}\right)\left(\geq \operatorname{ord}\left(s_{0}\right)+(1)\right) \geq \operatorname{ord}\left(r_{0}\right)-(m-1) .
$$

The inequality in parenthesis need not be true for all coordinates. It may be wrong for those coordinates, for which the corresponding inequality of $\operatorname{ord}\left(s_{0}\right)$ $\geq \operatorname{ord}\left(r_{0}\right)-(m)$ is proper. Nevertheless the inequality outside the parenthesis holds for all coordinates. From the decomposition $r^{\prime}=r+t s_{0}$ one obtains $r^{\prime}=r+t\left\langle A, r_{0}\right\rangle+t s_{0}^{\prime}$, hence

$$
r^{\prime \prime}:=\left\langle E-t A, r^{\prime}\right\rangle=r+t s_{0}^{\prime} .
$$

The matrix $E-t A$ is invertible, $(E-t A)(E+t A)=E$, so $r^{\prime \prime}$ is another lift of $\bar{r}$, whose coordinates generate $I^{\prime}$. Replacing the decomposition $r^{\prime}=r+t s_{0}$ by $r^{\prime \prime}=r+t s_{0}^{\prime}$, the integer $m$ decreases at least by one. So the proof of the implication (i) $\Rightarrow$ (ii) is reduced to the assertion that (8) gives a well-defined element of $N_{\operatorname{gr}\left(I_{0}, R_{0}\right)}$.

Let $H_{0} \in \operatorname{gr}\left(R_{0}\right)^{n}$ be such that $\left\langle H_{0}, \operatorname{in}\left(r_{0}\right)\right\rangle=0$. We have to show $\left\langle H_{0}, S_{0}\right\rangle \in$ $\operatorname{gr}\left(I_{0} R_{0}\right)$. For this we may assume the relation $\left\langle H_{0}, \operatorname{in}\left(r_{0}\right)\right\rangle=0$ is homogeneous, i.e.,

$$
\operatorname{deg}\left(H_{0}\right)+\operatorname{deg}\left(\operatorname{in}\left(r_{0}\right)\right)=(d)
$$

for some integer $d$. Since $\bar{R}$ is tangentially flat over $\bar{A}$, the relation $\left\langle H_{0}, \operatorname{in}\left(r_{0}\right)\right\rangle$ $=0$ in $\operatorname{gr}\left(R_{0}\right) \cong \operatorname{gr}(\bar{R}) /\left(\operatorname{gr}^{+}(\bar{A})\right)$ can be considered as a relation modulo $\left(\operatorname{gr}^{+}(\bar{A})\right)$ of $\operatorname{in}(\bar{r})$. Now $\operatorname{gr}(\bar{R}) / \operatorname{in}(\bar{r}) \operatorname{gr}(\bar{R})=\operatorname{gr}(\bar{B})$ is flat over $\operatorname{gr}(A)$, so this relation can be lifted to a relation of $\operatorname{in}(\bar{r})$ in $\operatorname{gr}(\bar{R})$. There is an $n$-tuple $H \in \operatorname{gr}(R)^{n}$ of homogeneous elements satisfying

$$
\langle H, \operatorname{in}(r)\rangle \equiv 0 \quad \bmod \operatorname{gr}(t R, R)
$$


and

$$
H_{0}=\left(H \bmod \operatorname{gr}^{+}(A) \operatorname{gr}(R)^{n}\right) .
$$

Choose $h \in R^{n}$ such that $\operatorname{in}(h)=H$. Then $\langle h, r\rangle \in \mathfrak{M}^{d+1}+t R$ and

$$
\operatorname{ord}(h)+\operatorname{ord}\left(r_{0}\right) \geq(d),
$$

the latter being a consequence of (9) and the fact that $\operatorname{ord}(r)=\operatorname{ord}\left(r_{0}\right)$. Since $\bar{r}$ is a standard base of $\bar{I}$, there is an $n$-tuple $h^{\prime} \in R^{n}$ such that

$$
\begin{gathered}
\langle h, r\rangle \equiv\left\langle h^{\prime}, r\right\rangle \quad \bmod t R, \\
\operatorname{ord}\left(h^{\prime}\right)+\operatorname{ord}\left(r_{0}\right) \geq(d+1) .
\end{gathered}
$$

Note that $\operatorname{ord}(r)=\operatorname{ord}\left(r_{0}\right)$. Let $x_{0} \in R_{0}$ be such that $\left\langle h-h^{\prime}, r\right\rangle=t x_{0}$. Then $\operatorname{ord}_{R}\left(t x_{0}\right)=\operatorname{ord}_{R}\left\langle h-h^{\prime}, r\right\rangle \geq d$ and by Theorem (1.5), since $R$ is tangentially flat over $A$,

$$
\operatorname{ord}_{R_{0}}\left(x_{0}\right) \geq d-e>d-m \text {. }
$$

From (11) we see,

$$
\left\langle h-h^{\prime}, r^{\prime}\right\rangle \equiv 0 \quad \bmod t R .
$$

Since $B^{\prime}=R / r^{\prime} R$ is flat over $A$, this relation modulo $t R$ lifts to a relation of $r^{\prime}$ in $R$. There exists some $y_{0} \in R_{0}^{n}$ such that

$$
\left\langle h-h^{\prime}+t y_{0}, r^{\prime}\right\rangle=0 .
$$

Indicating residue classes modulo $\mathfrak{m} R$ by subscripts " 0 ", we obtain

$$
\begin{aligned}
0 & =\left\langle h-h^{\prime}, r^{\prime}\right\rangle+t\left\langle y_{0}, r_{0}\right\rangle \\
& =\left\langle h-h^{\prime}, r\right\rangle+\left\langle h-h^{\prime}, t s_{0}\right\rangle+t\left\langle y_{0}, r_{0}\right\rangle \\
& =t\left(x_{0}+\left\langle h_{0}-h_{0}^{\prime}, s_{0}\right\rangle+\left\langle y_{0}, r_{0}\right\rangle\right),
\end{aligned}
$$

hence $0=x_{0}+\left\langle h_{0}-h_{0}^{\prime}, s_{0}\right\rangle+\left\langle y_{0}, r_{0}\right\rangle$ and

$$
\left\langle h_{0}, s_{0}\right\rangle-\left\langle h_{0}^{\prime}, s_{0}\right\rangle+x_{0} \in r_{0} R_{0}=I_{0} .
$$

We shall see that this implies the relation $\left\langle H_{0}, S_{0}\right\rangle \in \operatorname{gr}\left(I_{0}, R_{0}\right)$. To prove this, let us estimate the orders of the terms $\left\langle h_{0}, s_{0}\right\rangle,\left\langle h_{0}^{\prime}, s_{0}\right\rangle$, and $x_{0}$. Note that

$$
\begin{aligned}
\operatorname{ord}\left(h_{0}\right)+\operatorname{ord}\left(s_{0}\right) & \geq \operatorname{ord}\left(h_{0}\right)+\operatorname{ord}\left(r_{0}\right)-(m) \\
& \geq(d-m) \quad(\operatorname{by}(10)) \\
\operatorname{ord}\left(h_{0}^{\prime}\right)+\operatorname{ord}\left(s_{0}\right) & \geq \operatorname{ord}\left(h_{0}^{\prime}\right)+\operatorname{ord}\left(r_{0}\right)-(m) \\
& \geq(d+1-m) \quad(\operatorname{by}(11)) .
\end{aligned}
$$

Therefore

$$
\operatorname{ord}_{R_{0}}\left\langle h_{0}, s_{0}\right\rangle \geq d-m, \quad \operatorname{ord}_{R_{0}}\left\langle h_{0}^{\prime}, s_{0}\right\rangle>d-m, \quad \operatorname{ord}_{R_{0}} x_{0}>d-m
$$


(for the latter see (12)), hence

$$
\left\langle h_{0}, s_{0}\right\rangle+M_{0}^{d-m+1} \in \operatorname{gr}\left(I_{0}, R_{0}\right),
$$

which is just the relation $\left\langle H_{0}, S_{0}\right\rangle \in \operatorname{gr}\left(I_{0}, R_{0}\right)$ which was to be proved, for,

$$
H_{0}=\left(h_{0} \bmod \mathfrak{M}_{0}^{d-d(1)+1} \oplus \cdots \oplus \mathfrak{M}_{0}^{d-d(n)+1}\right) \quad(\text { see }(10))
$$

and

$$
S_{0}=\left(s_{0} \bmod \mathfrak{M}_{0}^{d(1)-m+1} \oplus \cdots \oplus \mathfrak{M}_{0}^{d(n)-m+1}\right) .
$$

(ii) $\Rightarrow$ (iii) Let $e:=\operatorname{ord}_{A}(t)$ be as above and assume that

$$
\operatorname{gr}\left(N_{I_{0}}\right)(<-e) \neq 0 \text {. }
$$

Take a nonzero element $g \in N_{I}=\operatorname{Hom}_{R_{0}}\left(I_{0}, R_{0} / I_{0}\right)$ with initial form of degree less than $-e$ in $\operatorname{gr}\left(N_{I_{0}}\right)$. Given a standard base $r_{0} \in R_{0}^{n}$ of $I_{0}$, there exists an $n$-tuple $s_{0} \in R_{0}^{n}$ such that

$$
g\left(\left\langle h_{0}, r_{0}\right\rangle\right)=\left\langle h_{0}, s_{0}\right\rangle \bmod I_{0}
$$

for every $h_{0} \in R_{0}^{n}$. By the choice of $g$,

$$
\operatorname{ord}_{R_{0} / I_{0}}\left(s_{0 i} \bmod I_{0}\right)<\operatorname{ord}_{R_{0}}\left(r_{0 i}\right)-e
$$

for at least one $i$. Here $s_{0 i}$ and $r_{0 i}$ denote the $i$ th coordinates of the vectors $s_{0}$ and $r_{0}$ respectively.

By assumption, $B=R / I$ is tangentially flat over $A$. So by Theorem (1.8) there is a lift $r$ of $r_{0}$ to a standard base of $I$ such that $\operatorname{ord}(r)=\operatorname{ord}\left(r_{0}\right)$. Define $r^{\prime}:=r+t s_{0}$ and let $I^{\prime}:=r^{\prime} R$ be the ideal of $R$ generated by the coordinates of $r^{\prime}$. Then $B^{\prime}:=R / I^{\prime}$ is flat over $A$. It is sufficient to show that $B^{\prime}$ is not tangentially flat. Assume the contrary and consider the coordinate $r_{i}^{\prime}=r_{i}+t s_{0}$ of $r^{\prime}$ with $i$ as in (13). The following identity holds in $B^{\prime}=R / I^{\prime}$ :

$$
t \cdot\left(s_{0 i} \bmod I_{0}\right)=-\left(r_{i} \bmod I^{\prime}\right) \text {. }
$$

Here $t$ is considered as an element of $B^{\prime}$. The term on the left is then a welldefined element of $B^{\prime}$, since $t$ annihilates $m$. By Theorem (1.5) this identity implies

$$
e+\operatorname{ord}_{R_{0} / I_{0}}\left(s_{0 i} \bmod I_{0}\right)=\operatorname{ord}_{B^{\prime}}\left(r_{i} \bmod I^{\prime}\right) \geq \operatorname{ord}_{R}\left(r_{i}\right)=\operatorname{ord}_{R_{0}}\left(r_{0 i}\right) .
$$

But this contradicts (13). Q.E.D.

(2.3) Definition. Let $(B, \mathfrak{n}, L)$ be a local ring. Its completion $\widehat{B}=R / I$ is a factor of a complete regular local ring $(R, \mathfrak{M}, L)$ modulo an ideal $I \subseteq \mathfrak{M}^{2}$. The module

$$
N_{B}:=N_{I}=\operatorname{Hom}_{R}(I, R / I)
$$

equipped with the filtration coming from the canonical filtration of $R$ will be called the normal module of $B$. Similarly,

$$
N_{\mathrm{gr}(B)}:=N_{\mathrm{gr}(I, R)}=\operatorname{Hom}_{\mathrm{gr}(R)}(\operatorname{gr}(I, R), \operatorname{gr}(R) / \operatorname{gr}(I, R))
$$


will be called the normal module of the tangent cone. Note that by the minimality condition $I \subseteq \mathfrak{M}^{2}, R$ and $I$ are determined up to (nonunique) isomorphism, and hence so are the modules $N_{B}, N_{\operatorname{gr}(B)}$, and $\operatorname{gr}\left(N_{B}\right)$.

The lemma below shows that, without the assumption $I \subseteq \mathfrak{M}^{2}, N_{\mathrm{gr}(B)}$ and $\operatorname{gr}\left(N_{B}\right)$ are unique up to direct factors isomorphic to $\operatorname{gr}(B)[1]$. Here as usual, $N[n]$ denotes, for any graded module $N=\bigoplus N(d)$, the graded module obtained from $N$ by shifting degrees, i.e., $N[n](d)=N(d+n)$.

(2.4) Lemma. (i) Let $J$ and $J^{\prime}$ be ideals of a ring $R$ such that $J=J^{\prime}+x R$ for some $x \in R$. Then $J^{\prime}: x \subseteq J$ if and only if

$$
J / J^{2}=\left(J^{\prime}+J^{2} / J^{2}\right) \oplus\left(x R+J^{2} / J^{2}\right)
$$

and multiplication by $x$ induces an isomorphism

$$
R / J \rightarrow x R+J^{2} / J^{2} \text {. }
$$

(ii) Let $S$ be a polynomial ring or a power series ring in one indeterminate $T$ over a Noetherian ring $R$, and let $I \subseteq R$ and $J \subseteq S$ be ideals such that $J=I S+F S$ for some $F \in S$ and such that the coefficients of $F$ generate the unit ideal in $R$. Then there is an isomorphism

$$
N_{J} \stackrel{\sim}{\rightarrow}\left(N_{I} \otimes_{R / I} S / J\right) \oplus S / J .
$$

In case $R$ is a graded ring and $F$ and $I$ are homogeneous, this is an isomorphism of graded $R$-modules

$$
N_{J} \stackrel{\sim}{\rightarrow}\left(N_{I} \otimes_{R / I} S / J\right) \oplus(S / J)[\operatorname{deg} F] .
$$

In case $(R, \mathfrak{M})$ is a local ring, $S=R[[T]]$, and $F=T$, the isomorphism (16) induces an isomorphism of graded $\operatorname{gr}(R)$-modules

$$
\operatorname{gr}\left(N_{J}\right) \stackrel{\sim}{\rightarrow} \operatorname{gr}\left(N_{I}\right) \oplus \operatorname{gr}(R / I)[1] .
$$

Proof. (i) Suppose conditions (14) and (15) are satisfied. Then

$$
R / J \stackrel{\sim}{\rightarrow} x R+J^{2} / J^{2} \cong\left(J / J^{2}\right) /\left(J^{\prime}+J^{2} / J^{2}\right)=J / J^{\prime}+J^{2},
$$

hence $a x \in J^{\prime}$ implies $a \in J$.

Conversely, assume that $J^{\prime}: x \subseteq J$. Then

$$
\left(J^{\prime}+J^{2}\right) \cap\left(x R+J^{2}\right)=J^{\prime} \cap(x R)+J^{2}=\left(J^{\prime}: x\right) x+J^{2}=J^{2} .
$$

This gives us the direct sum decomposition (14). Since (15) is obviously surjective, it is sufficient to prove it is injective. Let $a \in R$ represent an element of $R / J$, whose image is zero. Then $a x \in J^{2}=J^{\prime 2}+x J$. There is an element $a^{\prime} \in R$ such that $a^{\prime} x \in J^{\prime 2} \subseteq J^{\prime}$ and $a^{\prime} \equiv a \bmod J$. So our assumption implies $a^{\prime} \in J$, hence $a \in J$.

(ii) Note that the assumption on $F$ implies that the canonical homomorphism $R \rightarrow S / F S$ is flat and $F$ is $S / I S$-regular (see [Ma, Corollary of Theorem 22.6]). In particular, $I S: F=I S \subseteq J$, hence by the first part of the lemma,

$$
J / J^{2}=\left(I S+J^{2} / J^{2}\right) \oplus(S / J) .
$$


Note that

$$
\begin{aligned}
I S+J^{2} / J^{2} & \cong I S / I S \cap\left(I^{2}, I F, F^{2}\right) \\
& =I S /\left(I^{2}, I F\right)+(I) \cap\left(F^{2}\right)=I S /\left(I^{2}, I F\right) \\
& \cong I \otimes_{R} S / J \cong I / I^{2} \otimes_{R / I} S / J .
\end{aligned}
$$

So the above direct sum decomposition can be written

$$
J / J^{2} \cong\left[\left(I / I^{2}\right) \otimes_{R / I} S / J\right] \oplus(S / J) .
$$

Taking duals, one obtains

$$
\begin{aligned}
N_{J} & =\operatorname{Hom}_{S / J}\left(I / I^{2} \otimes_{R / I} S / J, S / J\right) \oplus(S / J) \\
& =\left[N_{I} \otimes_{R / I} S / J\right] \oplus(S / J),
\end{aligned}
$$

which is the first claim of (ii). We have used here that $S / J$ is flat over $R / I$ (see [Ma, Exercise (7.7)]).

In case $R$ is graded and $I$ and $F$ are homogeneous, multiplication by $F$ shifts degrees by $\operatorname{deg} F$. Taking this into account in the above calculations, one obtains the second claim of (ii).

Finally assume that $(R, \mathfrak{M})$ is a local ring, $S=R[[T]]$, and $F=T$. Then from the proof of (17) we see that the map

$$
I / I^{2} \oplus S / J \rightarrow J / J^{2}, \quad\left(a \bmod I^{2}, b \bmod J\right) \mapsto a+b T \bmod J^{2},
$$

is bijective. Note that the canonical map $R / I \rightarrow S / J$ is an isomorphism. In that what follows $R / I$ and $S / J$ will be identified. The bijection induces an isomorphism

$$
\begin{aligned}
N_{J}=\operatorname{Hom}_{S}(J, S / J) & \rightarrow \operatorname{Hom}_{R}(I, R / I) \oplus(R / I)=N_{I} \oplus(R / I), \\
f & \mapsto\left(\left.f\right|_{I}, f(T)\right) .
\end{aligned}
$$

By definition, $F_{N_{J}}^{d}$ consists of all $f \in N_{J}$ such that

$$
f\left(J \cap(\mathfrak{M}, T)^{k}\right) \subseteq(\mathfrak{M}, T)^{k+d}+J / J \cong \mathfrak{M}^{k+d}+I / I .
$$

Since

$$
\begin{aligned}
J \cap(\mathfrak{M}, T)^{k} & =\left(I+T R+T^{2} R+\cdots\right) \cap\left(\mathfrak{M}^{k}+\mathfrak{M}^{k-1} T+\mathfrak{M}^{k-2} G^{2}+\cdots\right) \\
& =I \cap \mathfrak{M}^{k}+\mathfrak{M}^{k-1} T+\mathfrak{M}^{k-2} T^{2}+\cdots,
\end{aligned}
$$

this condition is equivalent to

$$
f\left(I \cap \mathfrak{M}^{k}\right) \subseteq \mathfrak{M}^{k+d}+I / I \quad \text { and } \quad f(T) \in \mathfrak{M}^{d+1}+I / I .
$$

Therefore, the submodule $F_{N_{j}}^{d}$ of $N_{J}$ corresponds to the submodule

$$
F_{N_{l}}^{d} \oplus\left(\mathfrak{M}^{d+1}+I / I\right)
$$

of $N_{I} \oplus(R / I)$. This implies the last claim of (ii). Q.E.D. 
(2.5) Theorem. Let $\left(B_{0}, \mathfrak{n}_{0}\right)$ be a local ring containing a field. Consider the following conditions.

(i) $N_{\mathrm{gr}\left(B_{0}\right)}(<-1)=0$.

(ii) $\left(B_{0}, \mathfrak{n}_{0}\right)$ has only tangentially flat deformations.

(iii) $\operatorname{gr}\left(N_{B_{0}}\right)(<-1)=0$.

Then the implications (i) $\Rightarrow$ (ii) $\Rightarrow$ (iii) are true.

Proof. (i) $\Rightarrow$ (ii) Let $f:(A, \mathfrak{m}) \rightarrow(B, \mathfrak{n})$ be a flat local homomorphism such that $\widehat{B} / \mathrm{m} \widehat{B} \cong \widehat{B}_{0}$. We have to show that $f$ is tangentially flat. For this we may assume that $A, B$, and $B_{0}$ are complete local rings and by Remark (1.4)(ii) that $A$ is an Artin local ring. The further proof uses induction on $l:=$ length $(A)$.

In case $l=1$, the ring $A$ is a field and $f$ is obviously tangentially flat. Let $l>1$ and take some nonzero socle element $t \in A$. Then, by the induction hypothesis,

$$
\bar{f}:=f \otimes_{A}(A / t A): \bar{A} \rightarrow \bar{B}
$$

is tangentially flat. Since $f$ is flat and all rings are complete, there is, by Proposition (1.6), a commutative diagram of local homomorphisms

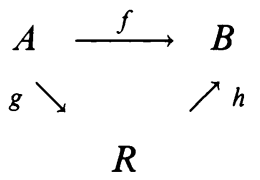

with $g$ tangentially flat and $h$ surjective. Let $I:=\operatorname{ker} h$, so that $B \cong R / I$. Using the notation of Proposition (2.2), we see from our hypothesis (i), that

$$
N_{\operatorname{gr}\left(I_{0}, R_{0}\right)}\left(<-\operatorname{ord}_{A}(t)\right) \subseteq N_{\operatorname{gr}\left(I_{0}, R_{0}\right)}(<-1)=0 .
$$

Hence, by the first implication of Proposition (2.2), the homomorphism $f: A \rightarrow$ $B$ is tangentially flat.

(ii) $\Rightarrow$ (iii) As above we may assume that $B_{0}$ is complete. Suppose condition (iii) is not satisfied. Define

$$
\begin{aligned}
& A:=K[t], \quad t^{2}=0, \quad t \notin K \\
& B:=B_{0}[t] \cong B_{0} \otimes_{K} A .
\end{aligned}
$$

Here $K$ is some field contained in $B_{0}$. By construction, the canonical homomorphism $f: A \rightarrow B$ is flat and $f \otimes_{A}(A / t A)$ is tangentially flat. As above there is a commutative diagram of local homomorphisms

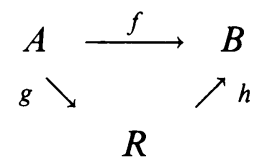

with $g$ tangentially flat and $h$ surjective. So Proposition (2.2) applies, proving that there must be some flat but not tangentially flat local homomorphism 
$A \rightarrow B^{\prime}$ with special fiber isomorphic to $B_{0}$. This is the desired contradiction. Q.E.D.

(2.6) Remarks. (i) In case $B_{0}$ is homogeneous, i.e., the completion $\widehat{B}_{0}$ is a factor of a power series ring over a field modulo an ideal generated by homogeneous polynomials, then

$$
\operatorname{gr}\left(N_{B_{0}}\right) \cong N_{\operatorname{gr}\left(B_{0}\right)}
$$

as graded modules. Conditions (i) and (iii) of the theorem are equivalent, and we have a necessary and sufficient condition in this case for a local ring to have only tangentially flat deformations.

(ii) It would be desirable to have the equivalence of the conditions of the theorem for more general situations, say for quasi-homogeneous singularities. The canonical filtration of $B_{0}$ (by powers of the maximal ideal) should then be replaced by some filtration more adapted to the concrete singularity, e.g. by some quasi-homogeneous filtration. Indeed, Theorem (2.5) and all the other assertions above have analogues with rather general filtrations instead of the canonical one. The treatment of the general case is much more technical and will be published elsewhere.

(iii) Condition (i) of the theorem can be found in a paper of J. Herzog (Essen) on what he calls strict local rings (see [He, Theorem 1]). The elementary Corollary (2.12) below just says that Artin local algebras over a field with only tangentially flat deformations are strict. Theorem 1 of $[\mathrm{He}]$ is a direct consequence of this fact and our Theorem (2.5).

Proof of (18). We may assume $B_{0}=R_{0} / I_{0}$ with $R_{0}$ a power series ring over some field and $I_{0}$ an ideal generated by homogeneous polynomials $f_{1}, \ldots, f_{n}$ and contained in the square of the maximal ideal. Then $f:=\left(f_{1}, \ldots, f_{n}\right)$ is a standard base of $I_{0}$ and its coordinates can be considered as a generating set of $\operatorname{gr}\left(I_{0}, R_{0}\right)$. The homogeneous elements of degree $d$ of $N_{\operatorname{gr}\left(B_{0}\right)}$ are given by $n$-tuples $g \in R_{0}^{n}$ of homogeneous elements of $R_{0}$ satisfying

$$
\langle r, f\rangle=0 \Rightarrow\langle r, g\rangle \in I_{0}, \quad \operatorname{deg} f+(d)=\operatorname{deg} g .
$$

Two such $n$-tuples $g, g^{\prime} \in R_{0}^{n}$ give the same element of $N_{\mathrm{gr}\left(B_{0}\right)}$ if and only if $g \equiv g^{\prime} \bmod I_{0} R_{0}^{n}$.

An analogous description of the elements of $N_{B_{0}}$ is obtained if one omits the second condition of (19) and the homogeneity condition. The elements of $F_{N_{B_{0}}}^{d}$ are given by $n$-tuples $g$ satisfying

$$
\operatorname{deg} f+(d) \leq \text { ord } g \text {. }
$$

From this it is easily seen that the elements of $\operatorname{gr}\left(N_{B_{0}}\right)(d)$ are given by $n$-tuples of homogeneous elements satisfying (19). Q.E.D. 
(2.7) Example. Let $B_{0}:=R_{0} / I_{0}$ and

$$
\begin{aligned}
& R_{0}:=L[[X, Y, Z]], \quad I_{0}:=\left(F_{1}, F_{2}, F_{3}, F_{4}, F_{5}, F_{6}\right), \\
& F_{1}:=X^{3}, \quad F_{2}:=Y^{3}, \quad F_{3}:=Z^{3}, \\
& F_{4}:=X^{2} Y, \quad F_{5}:=Y^{2} Z, \quad F_{6}:=Z^{2} X .
\end{aligned}
$$

Here $L$ denotes as usual some field. Then $B_{0}$ has only tangentially flat deformations.

Proof. We have to show $N_{\mathrm{gr}\left(B_{0}\right)}(<-1)=0$. The homogeneous elements of $N_{\mathrm{gr}\left(B_{0}\right)}$ of degree $d$ are given by 6-tuples $G$ of homogeneous polynomials $G_{1}, \ldots, G_{6}$ of degree $e=3+d$ such that

$$
\langle R, F\rangle=0 \text { implies }\langle R, G\rangle \in I_{0} .
$$

Here $F$ is the 6-tuple with coordinates $F_{1}, \ldots, F_{6}$. So we have to show that

\begin{tabular}{|c|c|c|c|c|c|c|}
\hline & $X^{3}$ & $Y^{3}$ & $Z^{3}$ & $X^{2} Y$ & $Y^{2} Z$ & $Z^{2} X$ \\
\hline & $Y$ & & & $\begin{array}{l}-X \\
Z^{2} \\
\end{array}$ & & $-X Y$ \\
\hline & $\begin{array}{l}X \\
0\end{array}$ & & & $\begin{array}{l}Y \\
0\end{array}$ & & 4 \\
\hline ymmetry: & & 0 & 0 & & 0 & 0 \\
\hline
\end{tabular}
any 6-tuple $G$ with homogeneous coordinates of degree $e=3+d<3-1=2$ satisfying (20) is the zero 6-tuple. The proof of this is concentrated in the following table.

The polynomials of the first row of the table are the generators $F_{i}$ of $I_{0}$. The space between the two horizontal lines contains the syzygies of these generators $F_{i}$ needed for the proof, and the space below indicates how to see that every $G_{i}$ must be zero.

Note that, since the $F_{i}$ 's are power products, we may assume that the $G_{i}$ 's are monomials (of equal degree $\leq 1$ ). From the first syzygy given in the table we see that $Y G_{1}-X G_{4} \in I_{0}$. Since $Y G_{1}-X G_{4}$ is a polynomial of degree at most two and $I_{0}$ is generated by power products of higher degree, this implies $Y G_{1}-X G_{4}=0$. In case $G_{1} \neq 0$ we may assume $G_{1}=X$ and $G_{4}=Y$. This is what we express by writing the indeterminates $X$ and $Y$ at the first row below the second line. From the second syzygy we see that $Z^{2} \cdot Y-X Y \cdot G_{6} \in I_{0}$. Since $I_{0}$ is generated by power products this implies either $Z^{2} \cdot Y, X Y G_{6} \in I_{0}$, or $Z^{2} \cdot Y-X Y \cdot G_{6}=0$. Both is obviously impossible, which we have indicated by writing " $y$ ". So $G_{1}$ must be zero, a fact that is expressed by the first figure " 0 " in the row above the last row. The first syzygy now implies $X G_{4} \in I_{0}$, hence $G_{4}=0$, which is the meaning of the second figure " 0 " in this row. By symmetry we conclude that the remaining coordinates must be zero, too, hence the figures " 0 " in the last row. Q.E.D. 
(2.8) Example. Let $B_{0}:=R_{0} / I_{0}$ and

$$
\begin{aligned}
& R_{0}:=L[[X, Y, Z]], \quad I_{0}:=\left(F_{1}, F_{2}, F_{3}\right), \\
& F_{1}:=X^{2}+Y Z, \quad F_{2}:=Y^{2}+X Z, \quad F_{3}:=X Y .
\end{aligned}
$$

Then $B_{0}$ has only tangentially flat deformations.

Proof. Using the conventions of the previous example, we have the following table.

$$
\begin{array}{ccc}
X^{2}+Y Z & Y^{2}+X Z & X Y \\
\hline X Z & -X^{2} & X Y-Z^{2} \\
\hline & 0
\end{array}
$$

0

Note that any $G \in R_{0}^{3}$ defining a homogeneous element of $N_{\operatorname{gr}\left(B_{0}\right)}$ of degree less than -1 has coordinates of degree zero. The figure " 0 " in the row above the last row arises, since $Z^{2}$ does not appear as a power product of some $F_{i}$. The remaining zeros come from the fact that

$$
X Z, \quad X^{2}, \quad F_{1}=X^{2}+Y Z, \quad F_{2}=Y^{2}+X Z, \quad F_{3}=X Y
$$

are linearly independent. Q.E.D.

(2.9) Example. Let $B_{0}:=R_{0} / I_{0}$ and

$$
\begin{aligned}
& R_{0}:=L[[X, Y, Z, W]], \quad I_{0}:=\left(F_{1}, F_{2}, F_{3}, F_{4}\right), \\
& F_{1}:=X W-Y Z, \quad F_{2}:=X^{2} Z-Y^{3}, \\
& F_{3}:=X Z^{2}-Y^{2} W, \quad F_{4}:=Y W^{2}-Z^{3} .
\end{aligned}
$$

Then $B_{0}$ has only tangentially flat deformations.

Note that $B_{0}$ is the completed local ring at the vertex of the affine cone over the Macaulay curve $V_{1,4}^{(2)}$ (the projection along the second coordinate axis of the Veronese variety $\mathbf{P}^{1} \cong V_{1,4} \hookrightarrow \mathbf{P}^{4}$ ).

Proof. The claim follows from the table below.

\begin{tabular}{cccc}
$X W-Y Z$ & $X^{2} Z-Y^{3}$ & $X Z^{2}-Y^{2} W$ & $Y W^{2}-Z^{3}$ \\
\hline$Y^{2}$ & $-Z$ & $X$ & \\
$Y W$ & & $-Z$ & $-X$ \\
$Z^{2}$ & & $-W$ & $-Y$ \\
$-X Z$ & $W$ & $-Y$ & \\
\hline 0 & $Y$ & $W$ & 4 \\
& 0 & 0 & 0
\end{tabular}

Q.E.D.

(2.10) Example. Let the local ring $\left(B_{0}, \mathfrak{n}_{0}\right)$ be a complete intersection, i.e., $\widehat{B}_{0} \cong R_{0} / I_{0}$ with

$$
R_{0}:=L\left[\left[X_{1}, \ldots, X_{N}\right]\right], \quad I_{0}:=\left(f_{1}, \ldots, f_{r}\right),
$$


and $f_{1}, \ldots, f_{r}$ a regular sequence in $R_{0}$. Suppose $B_{0}$ is not a regular local ring. Then $B_{0}$ has tangentially nonflat deformations.

Proof. We may assume $f_{1}$ has order $d_{1} \geq 2$ in $R_{0}$. There are isomorphisms

$$
\left(R_{0} / I_{0}\right)^{r} \stackrel{\sim}{\rightarrow} I_{0} / I_{0}^{2}, \quad\left(r_{i} \bmod I_{0}\right)_{i} \mapsto \sum_{i} r_{i} f_{i} \bmod I_{0}^{2}
$$

and

$$
N_{I_{0}} \stackrel{\sim}{\rightarrow}\left(R_{0} / I_{0}\right)^{r}, \quad g \mapsto\left(g\left(f_{1}\right), \ldots, g\left(f_{r}\right)\right) .
$$

The $r$-tuple $(1,0, \ldots, 0)$ of $\left(R_{0} / I_{0}\right)^{r}$ corresponds to the element

$$
g: I_{0} \rightarrow R_{0} / I_{0}, \quad \sum_{i} r_{i} f_{i} \mapsto r_{1} \quad \bmod I_{0}
$$

of $N_{I_{0}}$. The order of $g$ in $N_{I_{0}}$ is a most $-d_{1}$, since

$$
1=g\left(f_{1}\right) \in g\left(I_{0} \cap M_{0}^{d_{1}}\right) \nsubseteq M_{0} .
$$

Therefore

$$
0 \neq \operatorname{gr}\left(N_{I_{0}}\right)\left(\leq-d_{1}\right) \subseteq \operatorname{gr}\left(N_{I_{0}}\right)(<-1) \text {. Q.E.D. }
$$

(2.11) Example. Let $\left(B_{0}, \mathfrak{n}_{0}\right)$ be a local ring containing some field. Suppose there is some $B_{0}$-regular sequence $x_{1}, \ldots, x_{r} \in \mathfrak{n}_{0}$ such that $\bar{B}_{0}:=$ $B_{0} /\left(x_{1}, \ldots, x_{r}\right)$ has only tangentially flat deformations. Then the initial forms of the $x_{i}$ in $\operatorname{gr}\left(B_{0}\right)$ form a $\operatorname{gr}\left(B_{0}\right)$-regular sequence and $B_{0}$ has only tangentially flat deformations.

Proof. Let $f:(A, \mathfrak{m}) \rightarrow(B, \mathfrak{n})$ be a flat local homomorphism satisfying $\widehat{B} / \mathfrak{m} \widehat{B}$ $\cong \widehat{B}_{0}$. We want to show that $f$ is tangentially flat. For this we may assume that $A, B, B_{0}$ are complete local rings. Take elements $y_{i} \in B$ with residue classes in $B / \mathfrak{m} B \cong B_{0}$ corresponding to the $x_{i}$ 's. Then the sequence $y_{1}, \cdots, y_{r}$ is $B$-regular (see [Ma, Corollary to Theorem 22.5]). Since $B$ is complete, the mapping $f$ induces a local homomorphism

$$
A\left[\left[Y_{1}, \ldots, Y_{r}\right]\right] \rightarrow B
$$

sending the indeterminate $Y_{i}$ to $y_{i}$. Since the regular sequence $Y_{1}, \ldots, Y_{r}$ is mapped to a regular sequence in $B$, the homomorphism (21) is flat (see [ $\mathrm{Ma}$, Exercise (22.3)]). By construction, the special fiber of (21) is isomorphic to $\bar{B}_{0}$, i.e., (21) is tangentially flat. Composition with the canonical homomorphism $A \rightarrow A\left[\left[Y_{1}, \ldots, Y_{r}\right]\right]$, which is obviously tangentially flat, gives the homomorphism $f$. Hence $f$ is tangentially flat.

To prove the first part of the claim, let $K$ be some field contained in $B_{0}$ and consider the special case when $f$ is the canonical injection $K \rightarrow B_{0}$. As we have seen the associated homomorphism

$$
K\left[\left[Y_{1}, \ldots, Y_{r}\right]\right] \rightarrow B_{0}, \quad Y_{i} \mapsto x_{i},
$$


is tangentially flat, i.e.,

$$
K\left[Y_{1}, \ldots, Y_{r}\right] \rightarrow \operatorname{gr}\left(B_{0}\right), \quad Y_{i} \mapsto \operatorname{in}\left(x_{i}\right),
$$

is flat. Therefore the sequence of initial forms $\operatorname{in}\left(x_{1}\right), \ldots, \operatorname{in}\left(x_{r}\right)$ is $\operatorname{gr}\left(B_{0}\right)$ regular. Q.E.D.

(2.12) Corollary. Let $\left(B_{0}, \mathfrak{n}_{0}\right)$ be an Artin local ring containing some field and having only tangentially flat deformations. Then $\operatorname{gr}(B)$ is a Cohen-Macaulay ring for every local ring $B$ containing a $B$-sequence $x_{1}, \ldots, x_{r}$ such that

$$
B /\left(x_{1}, \ldots, x_{r}\right) \cong B_{0} \text {. }
$$

The example that follows is due to D. Eisenbud and J. Herzog (see [He, Proposition 1]) and gives a very useful criterion for a local ring to have only tangentially flat deformations. It implies that in the case of embedding dimension at least three all extremal Gorenstein algebras (see [Sche]) and many of the compressed Artin algebras (see [ I, (3.8) and (4.1A)]) have only tangentially flat deformations. (Originally the example was formulated for homogeneous ideals $I_{0}$ only, and the referee wondered whether the homogeneity hypothesis is needed. I want to thank him for this.)

(2.13) Example. Let $B_{0}=R_{0} / I_{0}$ be such that $R_{0}=L\left[\left[X_{1}, \ldots, X_{N}\right]\right]$ and $I_{0}$ has height at least two. Assume that the relation module of $\operatorname{gr}\left(I_{0}, R_{0}\right)$ is generated by linear syzygies. Then $B_{0}$ has only tangentially flat deformations.

Proof. See [He, Proposition 1].

\section{SINGULARITIES DEFINED BY POWER PRODUCTS}

(3.1) Proposition. Let $L$ be a field, $X=\left\{X_{1}, \ldots, X_{N}\right\}$ a finite set of indeterminates, and

$$
B_{0}:=L[[X]] /\left(s_{1}, \ldots, s_{n}\right)
$$

with power products $s_{1}, \ldots, s_{n}$ of equal degree $d$ such that every $s_{i}$ is contained in some subset $S(i) \subseteq\left\{s_{1}, \ldots, s_{n}\right\}$ satisfying the following conditions.

(i) The elements of $S(i)$ have greatest common divisor $\operatorname{GCD}(S(i))$ of degree at most one.

(ii) Any two elements $s, t \in S(i)$ can be connected by a sequence $s=$ $s_{i(0)}, s_{i(1)}, \ldots, s_{i(k)}=t$ of elements from $S(i)$ such that

$$
s_{i(j-1)} / s_{i(j)}=X_{u(j)} / X_{v(j)}
$$

is the quotient of two indeterminates for $j=1, \ldots, k$.

In this situation $B_{0}$ has only tangentially flat deformations.

(3.2) Definition. Let $S=\left\{s_{1}, \ldots, s_{n}\right\}$ be a set of power products of the indeterminates $X_{1}, \ldots, X_{N}$ such that any two elements $s, t \in S$ can be connected by a sequence $s=s_{i(0)}, s_{i(1)}, \ldots, s_{i(k)}=t$ of elements from $S$ such that 
$s_{i(j-1)} / s_{i(j)}=X_{u(j)} / X_{v(j)}$ is a quotient of two indeterminates for $j=1, \ldots, k$. Then we say that $S$ is 1-connected. Note that the elements of every 1-connected set of power products have equal degrees, so that it makes sense to speak of the degree of a 1-connected set. If $S$ is any set of power products, the maximal 1-connected subsets of $S$ will be called 1-components. With this terminology, Proposition (3.1) can be rephrased as follows.

(3.3) Let $B_{0}:=L\left[\left[X_{1}, \ldots, X_{N}\right]\right] /\left(s_{1}, \ldots, s_{n}\right)$ be such that $s_{1}, \ldots, s_{n}$ are power products of equal degree $d$ and such that each 1-component of ${ }^{n} S:=$ $\left\{s_{1}, \ldots, s_{n}\right\}$ has GCD of degree at most one. Then $B_{0}$ has only tangentially flat deformations.

(3.4) Remark. The set of generators $\left\{X^{3}, Y^{3}, Z^{3}, X^{2} Y, Y^{2} Z, Z^{2} X\right\}$ of the ideal $I_{0}$ of Example (2.7) consists of the three 1-components

$$
\left\{X^{2}, X^{2} Y\right\}, \quad\left\{Y^{3}, Y^{2} Z\right\}, \quad\left\{Z^{3}, Z^{2} X\right\}
$$

each having GCD of degree two. We shall see below that the conditions of Proposition (3.1) are far from being necessary even for power product ideals.

Proof of Proposition (3.1). Let $G_{1}, \ldots, G_{n} \in L[[X]]$ be homogeneous polynomials of degree $e<d-1$ such that $G:=\left(G_{1}, \ldots, G_{n}\right)$ defines an element of

$$
N:=N_{\mathrm{gr}\left(I_{0}, R_{0}\right)}, \quad I_{0}:=\left(s_{1}, \ldots, s_{n}\right) R_{0}, \quad R_{0}:=L[[X]] .
$$

We have to show $G$ defines the zero element of $N$, i.e.,

$$
G_{i} \in J_{0}:=\left(s_{1}, \ldots, s_{n}\right) L[X]
$$

for every $i$. Since the $s_{i}$ 's are power products, we may assume that the $G_{i}$ 's are monomials.

Consider the 1-component $S(i)$ of $S:=\left\{s_{1}, \ldots, s_{n}\right\}$ containing $s_{i}$, and take any sequence $s_{i(0)}, \ldots, s_{i(k)} \in S(i)$ as in condition (ii) of (3.1). From $X_{v(j)} s_{i(j-1)}-X_{u(j)} s_{i(j)}=0$ we see that

$$
X_{v(j)} G_{i(j-1)}-X_{u(j)} G_{i(j)} \in J_{0} .
$$

Since this polynomial has degree less than the degree $d$ of the generators of $J_{0}$, it must be zero:

$$
X_{v(j)} G_{i(j-1)}-X_{u(j)} G_{i(j)}=0 .
$$

Iterating this identity, we obtain $A G_{i(0)}=B G_{i(k)}$ with

$$
\begin{aligned}
A & :=\prod_{j=1}^{k} X_{v(j)} / \mathrm{GCD}\left(\prod_{j=1}^{k} X_{v(j)}, \prod_{j=1}^{k} X_{u(j)}\right), \\
B & :=\prod_{j=1}^{k} X_{u(j)} / \mathrm{GCD}\left(\prod_{j=1}^{k} X_{v(j)}, \prod_{j=1}^{k} X_{u(j)}\right) .
\end{aligned}
$$

Trivially we have the analogous identity $A s_{i(0)}=B s_{i(k)}$. 
Now consider the case $s_{i(0)}:=s_{i}:=X_{1}^{a(1)} \cdots X_{N}^{a(N)}$. By assumption there is at most one indeterminate, say $X_{j_{0}}$, dividing $\operatorname{GCD}(S(i))$. In case there is no such indeterminate let $X_{j_{0}}:=1$. Then, given some indeterminate $X_{j}$, the sequence $s_{i}=s_{i(0)}, s_{i(1)}, \ldots, s_{i(k)}$ can be chosen such that $X_{j} \nmid s_{i(k)}$ in case $j \neq j_{0}$ and such that $X_{j}^{2} \nmid s_{i(k)}$ in case $j=j_{0}$. From $A s_{i(0)}=B s_{i(k)}$ and $X_{j}^{a(j)} \mid s_{i(0)}$ we deduce $X_{j}^{a(j)} \mid B X_{j_{0}}$. Since $A G_{i(0)}=B G_{i(k)}$ and $A, B$ are relatively prime, this implies

$$
X_{j}^{a(j)} \mid G_{i(0)} X_{j_{0}}=G_{i} X_{j_{0}} .
$$

This last relation is true for arbitrary $j$, so that $s_{i} \mid G_{i} X_{j_{0}}$. Since the degree of $G_{i} X_{j_{0}}$ is less than $d=\operatorname{deg} s_{i}$, the $i$ th coordinate $G_{i}$ of $G$ must be zero. Q.E.D.

(3.5) Remark. Let $B_{0}:=L\left[\left[X_{1}, \ldots, X_{N}\right]\right] / I_{0}$ be such that $I_{0}$ is generated by power products $s_{1}, \ldots, s_{n}$ of degrees $\geq d$ and that there is a 1-connected subset $S$ of $\left\{s_{1}, \ldots, s_{n}\right\}$ of degree $d$ with GCD of degree at most one. Then the above argument shows that, given any homogeneous element

$$
f \in N_{\operatorname{gr}\left(B_{0}\right)}=\operatorname{Hom}\left(J_{0}, L[X] / J_{0}\right)
$$

of degree less than -1 , then $f(s)=0$ for $s \in S$.

(3.6) Proposition. Let $B_{0}:=L\left[\left[X_{1}, \ldots, X_{N}\right]\right] / I_{0}$ be such that $I_{0}$ is an ideal of $R_{0}:=L\left[\left[X_{1}, \ldots, X_{N}\right]\right]$ generated by power products $s_{1}, \ldots, s_{n}$ of degree two. Then the following are equivalent.

(i) $B_{0}$ has only tangentially flat deformations.

(ii) Every 1-component of $S:=\left\{s_{1}, \ldots, s_{n}\right\}$ has GCD of degree at most one, i.e., consists of at least two elements.

Proof. By (3.1) it suffices to show (i) implies (ii). Assume there is a 1-component of $S$ consisting of one single element, say $s_{1}$. Let $g:=(1,0, \ldots, 0) \in R_{0}^{n}$ be the first coordinate vector and $s:=\left(s_{1}, \ldots, s_{n}\right)$. It will be sufficient to show that $g$ defines an element of $N_{\operatorname{gr}\left(I_{0}, R_{0}\right)}$. Let $h \in R_{0}^{n}$ be such that $\langle h, s\rangle=0$. We have to prove $\langle h, g\rangle \in J_{0}:=\left(s_{1}, \ldots, s_{n}\right) L\left[X_{1}, \ldots, X_{N}\right]$. For this we may assume that $h$ is one of the generators of the module of syzygies of $s$. Since the coordinates of $s$ are monomials, we can take as generators the pairwise relations of the $s_{i}$ 's. The case that the first coordinate of $h$ vanishes is trivial, so we may assume $h$ comes from a relation of $s_{1}$ with some other $s_{i}$. By assumption, $s_{1}$ is relatively prime to all other elements of $S$, so $h$ is a multiple of $\left(s_{i}, 0, \ldots, 0,-s_{1}, 0, \ldots, 0\right)$, where all but the first and the $i$ th coordinate are zero. But then, $\langle h, g\rangle \in\left(s_{i}\right) \subseteq J_{0}$. Q.E.D.

The following two examples are directly derived from Proposition (3.1).

(3.7) Example. The local ring

$$
B_{0}:=L\left[\left[X_{1}, \ldots, X_{N}\right]\right] /\left(X_{1}, \ldots, X_{M}\right)^{d}
$$

satisfying $2 \leq M \leq N$ has only tangentially flat deformations. 
(3.8) Remark. Let $K$ be some field and $\left(B_{0}, \mathfrak{n}_{0}\right)$ some local $K$-algebra with only tangentially flat deformations. From $(2.11)$ we know that every local $K$ algebra $\left(B_{0}^{\prime}, \mathfrak{n}_{0}^{\prime}\right)$ such that

$$
B_{0}^{\prime} /\left(x_{1}, \ldots, x_{r}\right)=B_{0}
$$

for some regular sequence $x_{1}, \ldots, x_{r}$ is of the same kind. So, starting with $B_{0}$, one can produce a whole series of singularities $B_{0}^{\prime}$ with only tangentially flat deformations. Applied to Example (3.7) the algebras $B_{0}^{\prime}$ obtained in this way are just the so-called extremal Cohen-Macaulay algebras (more precisely those algebras for which $\operatorname{gr}\left(B_{0}^{\prime}\right)$ is extremal Cohen-Macaulay, see [Sche]). Among them are, for example, the singularities defined by the maximal minors of generic matrices and the Cohen-Macaulay singularities of maximal embedding dimension.

(3.9) Example. The local ring

$$
B_{0}:=L\left[\left[X_{1}, \ldots, X_{N}\right]\right] / X_{1}\left(X_{1}, \ldots, X_{M}\right)^{d}
$$

satisfying $2 \leq M \leq N$ has only tangentially flat deformations.

(3.10) Proposition. Let $X$ and $Y$ be two indeterminates and let

$$
B_{0}:=L[[X, Y]] / I_{0}
$$

be such that the ideal $I_{0}$ is generated by power products. Then the following are equivalent.

(i) $B_{0}$ has only tangentially flat deformations.

(ii) $I_{0}$ is one of the ideals $(X, Y)^{d}, X(X, Y)^{d-1}, Y(X, Y)^{d-1}(d=$ $1,2, \ldots)$.

Proof. By (3.7) and (3.9) it is sufficient to show that (i) implies (ii). Let $s_{0}, \ldots, s_{n} \in R_{0}:=L[[X, Y]]$ be power products forming a minimal generating set of $I_{0}$. Write

$$
s_{i}=X^{a(i)} Y^{b(i)}, \quad i=0, \ldots, n,
$$

and assume that the $s_{i}$ 's are arranged such that $a(0)>a(1)>\cdots>a(n)$. Then $b(0)<b(1)<\cdots<b(n)$, since otherwise some $s_{i}$ could be omitted. The relation module of $s_{0}, \ldots, s_{n}$ is generated by the syzygies

$$
Y^{d(i)} s_{i-1}-X^{e(i)} s_{i}=0, \quad i=1, \ldots, n .
$$

Here $d(i):=b(i)-b(i-1)$ and $e(i):=a(i-1)-a(i)$. First consider the case that $d(i)=e(i)=1$ for every $i$. Then $s_{i}=X^{a-i} Y^{b+i}$ for $i=0, \ldots, n$ and suitable $a, b$. The set $S:=\left\{s_{0}, \ldots, s_{n}\right\}$ is 1 -connected with GCD equal to $D:=X^{a-n} Y^{b}$. Define

$$
s:=\left(s_{0}, \ldots, s_{n}\right), \quad g:=\left(g_{0}, \ldots, g_{n}\right), \quad g_{i}:=s_{i} / D .
$$


Then $\langle r, s\rangle=0$ implies $\langle r, g\rangle=0 \in \operatorname{gr}\left(I_{0}, R_{0}\right)$, i.e., $g$ defines an element of $N_{\mathrm{gr}\left(I_{0}, R_{0}\right)}$. Since by assumption $N_{\mathrm{gr}\left(I_{0}, R_{0}\right)}(<-1)=0$,

$$
-\operatorname{deg} D=\operatorname{deg} g_{i}-\operatorname{deg} s_{i} \geq-1,
$$

i.e., $\operatorname{deg} D \leq 1$. Hence either $D=1, D=X$, or $D=Y$. So $I_{0}$ is one of the ideals of (ii).

Next consider the remaining case $d(r) \geq 2$ for some $r$. It suffices to show that there is a tangentially nonflat deformation of $B_{0}$. Define

$$
t_{i}:= \begin{cases}s_{i} & \text { for } i=1, \ldots, r-1, \\ \left(1+Z / Y^{d(r)}\right) s_{i} & \text { for } i=r, \ldots, n\end{cases}
$$

with $Z$ one further indeterminate and let

$$
A:=L[[Z]], \quad B:=A[[X, Y]] /\left(t_{0}, \ldots, t_{n}\right) .
$$

Note that $s_{r}, \ldots, s_{n}$ are multiples of $Y^{d(r)}$. To prove that $B$ is flat over $A$ it is sufficient to show that the relations (22), which can be considered as relations modulo $(Z)$ of the $t_{i}$ 's, come from relations of the $t_{i}$ 's in $A[[X, Y]]$.

Case 1. Assume $i<r$. Then

$$
Y^{d(i)} t_{i-1}-X^{e(i)} t_{i}=Y^{d(i)} s_{i-1}-X^{e(1)} s_{i}=0 .
$$

Case 2. Assume $i=r$. Then

$$
\left(Y^{d(r)}+Z\right) t_{r-1}-X^{e(r)} t_{r}=\left(1+\frac{Z}{Y^{d(r)}}\right)\left(Y^{d(r)} s_{r-1}-X^{e(r)} s_{r}\right)=0 .
$$

Case 3. Assume $i>r$. Then

$$
Y^{d(i)} t_{i-1}-X^{e(i)} t_{i}=\left(1+\frac{Z}{Y^{d(r)}}\right)\left(Y^{d(i)} s_{i-1}-X^{e(i)} s_{i}\right)=0 .
$$

We have proved that $B$ is flat over $A$. For any $b \in A[[X, Y]]$ let $\bar{b}$ denote the residue class of $b$ in $B$. Then

$$
\operatorname{ord}_{B}\left(\bar{Z} \cdot \overline{s_{r} / Y^{d(r)}}\right)=\operatorname{ord}_{B}\left(-\bar{s}_{r}\right) \geq \operatorname{deg} s_{r} .
$$

Note that the residue class of $s_{r} / Y^{d(r)}$ in the special fiber of $B$ over $A$ has order

$$
\operatorname{deg} s_{r} / Y^{d(r)}=\operatorname{ord}_{B} \overline{\left(s_{r} / Y^{d(r)}\right)} \text {. }
$$

By Theorem (1.5), tangential flatness of $B$ over $A$ would imply

$$
\operatorname{ord}_{A}(Z) \geq \operatorname{deg} s_{r}-\operatorname{ord}_{B}\left(s_{r} / Y^{d(r)}\right)=d(r) \geq 2,
$$

which is absurd. Q.E.D.

(3.11) Example. The local ring

$$
B_{0}:=L\left[\left[X_{1}, \ldots, X_{N}\right]\right] /\left(X_{2}, \ldots, X_{N}\right)^{d}+X_{1}\left(X_{2}, \ldots, X_{N}\right)^{d-2}
$$

with $N \geq 3, d \geq 2$ has only tangentially flat deformations. 
Proof. Let $s_{1}^{0}, \ldots, s_{n}^{0}$ and $s_{1}^{1}, \ldots, s_{m}^{1}$ be the power products of $X_{2}, \ldots, X_{N}$ of degree $d$ and $d-2$ respectively. Then the ideal $I_{0}:=\left(X_{2}, \ldots, X_{N}\right)^{d}+$ $X_{1}\left(X_{2}, \ldots, X_{N}\right)^{d-2}$ is generated by the family

$$
t:=\left(t_{j}^{i}\right), \quad t_{j}^{i}:=X_{1}^{i} s_{j}^{i}, \quad i=0,1 .
$$

Let $g=\left(g_{j}^{i}\right)$ be a family of power products defining an element of $N_{\operatorname{gr}\left(I_{0}, R_{0}\right)}$ such that

$$
\operatorname{deg} g_{j}^{i}-\operatorname{deg} t_{j}^{i}=e<-1 .
$$

We have to show that the element of $N_{\operatorname{gr}\left(I_{0}, R_{0}\right)}$ defined by $g$ is zero. Note that the set of $t_{j}^{i}$ 's consists of the two 1-components $\left\{t_{1}^{0}, \ldots, t_{n}^{0}\right\}$ and $\left\{t_{1}^{1}, \ldots, t_{m}^{1}\right\}$ of degree $d$ and $d-1$ respectively. From Remark (3.5) we see that $g_{j}^{1} \in$ $\operatorname{gr}\left(I_{0}, R_{0}\right)$ for every $j$, hence we may assume $g_{j}^{1}=0$ for every $j$.

For arbitrary $i$ the monomial $X_{1} t_{i}^{0}$ is divisible by some $t_{j}^{1}$, i.e.,

$$
X_{1} t_{i}^{0}-X^{a} t_{j}^{1}=0
$$

for some power product $X^{a}$ of degree two depending upon $i$. Therefore

$$
X_{1} g_{i}^{0}=X_{1} g_{i}^{0}-X^{a} g_{j}^{1} \in \operatorname{gr}\left(I_{0}, R_{0}\right) .
$$

The degree of $X_{1} g_{i}^{0}$ is less than $d$, so $X_{1} g_{i}^{0} \in X_{1}\left(X_{2}, \ldots, X_{N}\right)^{d-2}$, hence $g_{i}^{0} \in\left(X_{2}, \ldots, X_{N}\right)^{d-2}$ for every $i$.

Next, let $t_{i}^{0}$ and $t_{i^{\prime}}^{0}$ be such that $t_{i}^{0} / t_{i^{\prime}}^{0}$ is a quotient of two indeterminates, say

$$
X_{v} t_{i}^{0}-X_{u} t_{i^{\prime}}^{0}=0, \quad u, v \neq 1 .
$$

Then $X_{v} g_{i}^{0}-X_{u} g_{i^{\prime}}^{0} \in \operatorname{gr}\left(I_{0}, R_{0}\right)$. Since $X_{v} g_{i}^{0}$ and $X_{u} g_{i^{\prime}}^{0}$ have degrees less than $d$, their difference is even in $X_{1}\left(X_{2}, \ldots, X_{N}\right)^{d-2}$, i.e., $X_{v} g_{i}^{0}-X_{u} g_{i^{\prime}}^{0} \equiv 0$ $\bmod \left(X_{1}\right)$. Hence modulo $X_{1}$,

$$
g_{i}^{0}: g_{i^{\prime}}^{0} \equiv X_{u}: X_{v}=s_{i}^{0}: s_{i^{\prime}}^{0} .
$$

The vector $g^{0}:=\left(g_{1}^{0}, \ldots, g_{n}^{0}\right)$ is proportional modulo $X_{1}$ to the vector $s^{0}:=$ $\left(s_{1}^{0}, \ldots, s_{n}^{0}\right)$. Since $g^{0}$ has coordinates of degree less than $d$, this implies $g_{i}^{0} \equiv 0 \bmod \left(X_{1}\right)$ for every $i$.

On the other hand we have proved $g_{i}^{0} \in\left(X_{2}, \ldots, X_{N}\right)^{d-2}$. Therefore, $g_{i}^{0} \in$ $X_{1}\left(X_{2}, \ldots, X_{N}\right)^{d-2}$ and, observing that $\operatorname{deg} g_{i}^{0}<d-1, g_{i}^{0}=0$. Q.E.D.

(3.12) Theorem. Let $L$ be a field and $I_{0}$ an ideal of the power series ring $R_{0}:=L[[X, Y, Z]]$ generated by power products $s_{1}, \ldots, s_{n}$ of degree two and three. Suppose that $S:=\left\{s_{1}, \ldots, s_{n}\right\}$ is a minimal generating set for $I_{0}$ and that $\operatorname{dim} B_{0}=0$. 
Then $B_{0}:=R_{0} / I_{0}$ has only tangentially flat deformations if and only if $S$ is, up to permutation of the indeterminates, one of the sets enumerated below.

\begin{tabular}{|c|c|c|c|c|c|}
\hline & elements of $S$ & $e_{0}\left(B_{0}\right)$ & $H_{B_{0}}^{0}$ & \multicolumn{2}{|c|}{$\operatorname{dim}_{L} T_{B_{0}}^{1}$} \\
\hline 1. & $\begin{array}{l}X^{2}, X Y, X Z, Y^{2}, Y Z \\
Z^{2}\end{array}$ & 4 & $1+3 T$ & 15 & \\
\hline \multirow[t]{2}{*}{2.} & $X^{2}, X Y, X Z, Y^{2}, Z^{2}$ & 5 & $1+3 T+T^{2}$ & 8 & $c \neq 2$ \\
\hline & & & & 10 & $c=2$ \\
\hline \multirow[t]{2}{*}{3.} & $X^{2}, X Z, Y^{2}, Y Z^{2}, Z^{3}$ & 7 & $1+3 T+3 T^{2}$ & 13 & $c \neq 2$ \\
\hline & & & & 15 & $c=2$ \\
\hline \multirow[t]{2}{*}{4.} & $X^{2}, Y^{2}, Z^{2}, X Y Z$ & 7 & $1+3 T+3 T^{2}$ & 12, & $c \neq 2$ \\
\hline & & & & 18 & $c=2$ \\
\hline \multirow[t]{2}{*}{5.} & $X^{2}, Z^{2}, X Y^{2}, X Y Z$ & 8 & $1+3 T+4 T^{2}$ & 23 & $c \neq 2$ \\
\hline & $Y^{3}, Y^{2} Z$ & & & 27 , & $c=2$ \\
\hline \multirow[t]{2}{*}{6.} & $X Z, Y^{2}, X^{3}, X^{2} Y$ & 8 & $1+3 T+4 T^{2}$ & 21, & $c \neq 2$ \\
\hline & $Y Z^{2}, Z^{3}$ & & & 23 & $c=2$ \\
\hline \multirow[t]{2}{*}{7.} & $X^{2}, X Y^{2}, X Y Z, X Z^{2}$ & 9 & $1+3 T+5 T^{2}$ & 38, & $c \neq 2$ \\
\hline & $Y^{3}, Y^{2} Z, Y Z^{2}, Z^{3}$ & & & 40 & $c=2$ \\
\hline \multirow[t]{2}{*}{8.} & $X Z, X^{3}, X^{2} Y, X Y^{2}$ & 9 & $1+3 T+5 T^{2}$ & 36 & \\
\hline & $Y^{3}, Y^{2} Z, Y Z^{2}, Z^{3}$ & & & & \\
\hline \multirow[t]{2}{*}{9.} & $X^{2}, X Y^{2}, X Z^{2}, Y^{3}$ & 10 & $1+3 T+5 T^{2}+T^{3}$ & 26 , & $c \neq 2$ \\
\hline & $Y^{2} Z, Y Z^{2}, Z^{3}$ & & & 33 & $c=2$ \\
\hline \multirow[t]{3}{*}{10.} & $X^{2}, X Y^{2}, X Y Z, Y^{3}$ & 10 & $1+3 T+5 T^{2}+T^{3}$ & 27 & $c \neq 2,3$ \\
\hline & $Y^{2} Z, Y Z^{2}, Z^{3}$ & & & 28 & $c=3$ \\
\hline & & & & 30 , & $c=2$ \\
\hline
\end{tabular}

11. $X^{3}, X^{2} Y, X^{2} Z, X Y^{2}$,

$X Y Z, X Z^{2}, Y^{3}, Y^{2} Z$,

$101+3 T+6 T^{2}$

57

$Y Z^{2}, Z^{3}$

12. $X^{2}, X Y^{2}, X Y Z, Y^{3}$

$11 \quad 1+3 T+5 T^{2}+2 T^{3} \quad 23, \quad c \neq 2,3$

$Y Z^{2}, Z^{3}$

$24, c=3$

$25, c=2$

13. $X^{2}, X Y Z, Y^{3}, Y^{2}, Z$,

$11 \quad 1+3 T+5 T^{2}+2 T^{3} \quad 22, \quad c \neq 2$

$Y Z^{2}, Z^{3}$

$26, c=2$

14. $X^{2}, X Z^{2}, Y^{3}, Y^{2} Z$,

$11 \quad 1+3 T+5 T^{2}+2 T^{3} \quad 23, \quad c \neq 2$

$Y Z^{2}, Z^{3}$

$28, c=2$

15. $X^{3}, X^{2} Y, X^{2} Z, X Y^{2}$,

$X Z^{2}, Y^{3}, Y^{2} Z, Y Z^{2}$,

$11 \quad 1+3 T+6 T^{2}+T^{3}$

$42, \quad c \neq 2$

$Z^{3}$

$48, \quad c=2$ 


\begin{tabular}{|c|c|c|c|c|c|}
\hline & elements of $S$ & $e_{0}\left(B_{0}\right)$ & $H_{B_{0}}^{0}$ & \multicolumn{2}{|c|}{$\operatorname{dim}_{L} T_{B_{0}}^{1}$} \\
\hline \multirow[t]{3}{*}{16.} & $X^{3}, X^{2} Y, X^{2} Z, X Y Z$ & & & & \\
\hline & $X Z^{2}, Y^{3}, Y^{2} Z, Y Z^{2}$ & 11 & $1+3 T+6 T^{2}+T^{3}$ & 44, & $c \neq 2,3$ \\
\hline & $Z^{3}$ & & & 46, & $c=2,3$ \\
\hline \multirow[t]{2}{*}{17.} & $X^{3}, X^{2} Y, X^{2} Z, X Z^{2}$ & 12 & $1+3 T+6 T^{2}+2 T^{3}$ & 38 & $c \neq 2$ \\
\hline & $Y^{3}, Y^{2} Z, Y Z^{2}, Z^{3}$ & & & 41, & $c=2$ \\
\hline \multirow[t]{2}{*}{18.} & $X^{3}, X^{2} Z, X Y^{2}, X Y Z$ & 12 & $1+3 T+6 T^{2}+2 T^{3}$ & 37 , & $c \neq 2$ \\
\hline & $X Z^{2}, Y^{3}, Y^{2} Z, Z^{3}$ & & & 39 & $c=2$ \\
\hline \multirow[t]{2}{*}{19.} & $X^{3}, X^{2} Z, X Y^{2}, X Y Z$ & 12 & $1+3 T+6 T^{2}+2 T^{3}$ & 37 & $c \neq 2,3$ \\
\hline & $X Z^{2}, Y^{3}, Y Z^{2}, Z^{3}$ & & & 38 & $c=2,3$ \\
\hline \multirow[t]{2}{*}{20.} & $X^{3}, X^{2} Z, X Y^{2}, X Y Z$ & 13 & $1+3 T+6 T^{2}+2 T^{3}$ & 36 & \\
\hline & $Y^{3}, Y Z^{2}, Z^{3}$ & & & & \\
\hline \multirow[t]{2}{*}{21.} & $X^{3}, X^{2} Z, X Y Z, X Z^{2}$ & 13 & $1+3 T+6 T^{2}+2 T^{3}+T^{4}$ & 33 & $c \neq 3$ \\
\hline & $Y^{3}, Y^{2} Z, Y Z^{2}, Z^{3}$ & & & 37, & $c=3$ \\
\hline \multirow[t]{3}{*}{22.} & $X^{3}, X^{2} Z, X Y Z, X Z^{2}$ & 14 & $1+3 T+6 T^{2}+3 T^{3}+T^{4}$ & 28, & $c \neq 2,3$ \\
\hline & $Y^{3}, Y^{2} Z, Z^{3}$ & & & 29 , & $c=2$ \\
\hline & & & & 31, & $c=3$ \\
\hline \multirow[t]{2}{*}{23.} & $X^{3}, X^{2} Z, X Z^{2}, Y^{3}$ & 14 & $1+3 T+6 T^{2}+3 T^{3}+T^{4}$ & 31, & $c \neq 3$ \\
\hline & $Y^{2} Z, Y Z^{2}, Z^{3}$ & & & 33 & $c=3$ \\
\hline \multirow[t]{2}{*}{24.} & $X^{3}, X^{2} Z, X Y^{2}, Y^{3}$ & 14 & $1+3 T+6 T^{2}+4 T^{3}$ & 39 & \\
\hline & $X Z^{2}, Z^{3}$ & & & & \\
\hline
\end{tabular}

In the table $e_{0}\left(B_{0}\right)$ denotes the multiplicity of $B_{0}, T_{B_{0}}^{1}$ is Schlessinger's module, and $c$ is the characteristic of $L$.

The proof of the theorem is tedious and consists in checking a rather large number of cases using the previous propositions and the method of Example (2.7). We omit it.

\section{FURTHER EXAMPLES}

In this section we use the results obtained so far to construct singularities with only tangentially flat deformations, which are not necessarily defined by power products. The following example illustrates how to decide whether an inhomogeneous singularity has only tangentially flat deformations. One uses the fact that, by Theorem (2.5), the existence of tangentially nonflat deformations implies the existence of such deformations for the associated singularity defined by homogeneous equations (or by power products, see below).

(4.1) Example. Let the ideal $I_{0}$ of $R_{0}:=L[[X, Y, Z]]$ be generated by the 
polynomials

$$
f_{1}:=X^{2}+Z^{3}, \quad f_{2}:=X Y Z, \quad f_{3}:=Z^{2}+X^{3} .
$$

Then $B_{0}:=R_{0} / I_{0}$ has only tangentially flat deformations.

Proof. Let $f_{10}:=X^{2}, f_{20}:=X Y Z, f_{30}:=Z^{2}$ be the initial forms of the $f_{i}$. We first show that the ideal $I_{0}^{\prime}$ generated by $f_{10}, f_{20}, f_{30}$ defines a singularity with only tangentially flat deformations. This is easy, since there is no problem to write down the syzygies of the $f_{i 0}$ 's, and follows from the table below (using the terminology of Example (2.7)).

\begin{tabular}{lccc}
$r_{10}$ & $X^{2}$ & $X Y Z$ & $Z^{2}$ \\
\cline { 2 - 4 } & $Y Z$ & $-X$ & \\
$r_{20}$ & $Z$ & $-X Y$ \\
$r_{30}$ & $Z^{2}$ & & $-X^{2}$ \\
\cline { 2 - 4 } & 0 & 0 & 0
\end{tabular}

Note that the syzygies of the table constitute a complete set of generators for the relation module of $f_{0}:=\left(f_{10}, f_{20}, f_{30}\right)$. Further, all these syzygies come from syzygies of $f:=\left(f_{1}, f_{2}, f_{3}\right)$ :

$$
\begin{aligned}
& \left\langle r_{10}, f\right\rangle=Y Z^{4}=-X^{2} Z f_{2}+Y Z^{2} f_{3}, \\
& \left\langle r_{20}, f\right\rangle=-X^{4} Y=-X^{2} Y f_{1}+X Z^{2} f_{2}, \\
& \left\langle r_{30}, f\right\rangle=Z^{5}-X^{5}=-X^{3} f_{1}+Z^{3} f_{3} .
\end{aligned}
$$

So the (well-known) Lemma (4.3) below implies that the initial ideal of $I_{0}$ is generated by $f_{10}, f_{20}, f_{30}$, i.e.,

$$
\operatorname{gr}\left(I_{0}, R_{0}\right)=\operatorname{gr}\left(I_{0}^{\prime}, R_{0}\right) .
$$

Therefore,

$$
N_{\operatorname{gr}\left(I_{0}, R_{0}\right)}(<-1)=N_{\mathrm{gr}\left(I_{0}^{\prime}, R_{0}\right)}(<-1)=0,
$$

and the claim follows from Theorem (2.5). Q.E.D.

To state the lemma in the context general enough for later use, we have to introduce some terminology.

(4.2) Definition. Let $L$ be a field and $X_{1}, \ldots, X_{N}$ finitely many indeterminates. An order function on the power products in $X_{1}, \ldots, X_{N}$ is a map

$$
\text { ord: }\left\{X^{v} \mid v \in \mathbf{N}^{N}\right\} \rightarrow \mathbf{N}
$$

associating to each power product $X^{v}=X_{1}^{v(1)} \cdots X_{N}^{v(N)}$ a nonnegative integer $\operatorname{ord}\left(X^{v}\right)$ and satisfying the following conditions.

(i) $\operatorname{ord}\left(X^{v}\right) \leq \operatorname{ord}\left(X^{w}\right)$ if $X^{v}$ divides $X^{w}$.

(ii) Given any $d$, there are only finitely many power products $X^{v}$ such that $\operatorname{ord}\left(X^{v}\right)=d$. 
Note that the sequence

$$
\left\{\min \left\{\operatorname{deg}\left(X^{v}\right) \mid \operatorname{ord}\left(X^{v}\right) \geq d\right\}\right\}_{d=0,1,2, \ldots}
$$

goes to infinity.

The standard example for an order function is

$$
\operatorname{deg}\left(X^{v}\right)=|v|:=v(1)+\cdots+v(N)
$$

associating to each power product its total degree. Another example is the lexicographic order function

$$
\operatorname{Ord}\left(X^{v}\right):=\left\{\begin{array}{l}
\text { number of power products } \\
\text { preceding } X^{v} \text { lexicographically }
\end{array}\right.
$$

(order first by total degree and then, within each degree, lexicographically). Further examples are the weights associated with graded Hodge algebras (see [DEP, proof of Proposition 1.1]).

The filtration of $R_{0}:=L\left[\left[X_{1}, \ldots, X_{N}\right]\right]$ associated with the order function is defined by

$$
F^{d} R_{0}:=\left(X^{v} \mid \operatorname{ord}\left(X^{v}\right) \geq d\right) R_{0}
$$

for $d \in \mathbf{N}$. Let $I_{0}$ be an ideal of $R_{0}$ and $\bar{F} B_{0}$ the filtration induced by $F R_{0}=\left\{F^{d} R_{0}\right\}$ on $B_{0}:=R_{0} / I_{0}$ (i.e., $F^{d} B_{0}$ is the canonical image of $F^{d} R_{0}$ in $B_{0}$ ). Further let

$$
\alpha: \mathbf{N} \times \mathbf{N} \rightarrow \mathbf{N}
$$

define a commutative semigroup structure on the nonnegative integers with $0 \in$ $\mathbf{N}$ the identity element such that $\alpha$ is strictly monotonous with respect to both variables and such that

$$
F^{d} R_{0} \cdot F^{d^{\prime}} R_{0} \subseteq F^{\alpha\left(d, d^{\prime}\right)} R_{0}
$$

for $d, d^{\prime} \in \mathbf{N}$. We shall say in this case that $\alpha$ is compatible with ord.

The function $\alpha$ can be the usual addition,

$$
\alpha\left(d, d^{\prime}\right)=d+d^{\prime},
$$

in case ord is the weight coming from some Hodge algebra structure on $B_{0}$ or in case ord $=\mathrm{deg}$. In case ord $=$ Ord is the lexicographic order, $\alpha$ can be chosen to be

$$
\alpha\left(d, d^{\prime}\right):=\min \left\{\operatorname{Ord}\left(X^{v+v^{\prime}}\right) \mid \operatorname{Ord}\left(X^{v}\right) \geq d, \operatorname{Ord}\left(X^{v^{\prime}}\right) \geq d^{\prime}\right\} .
$$

Given any such $\alpha$, the graded module

$$
\operatorname{gr}_{F, \alpha}\left(B_{0}\right):=\bigoplus_{d \geq 0} \bar{F}^{d} B_{0} / \bar{F}^{d+1} B_{0}
$$

associated with the filtration $F R_{0}$ admits an $(\mathbf{N}, \alpha)$-graded ring structure such that

$$
\begin{gathered}
\left(r \bmod \bar{F}^{d+1} B_{0}\right) \cdot\left(r^{\prime} \bmod \bar{F}^{d^{\prime}+1} B_{0}\right) \\
=\left(r \cdot r^{\prime} \bmod \bar{F}^{\alpha\left(d, d^{\prime}\right)+1} B_{0}\right) .
\end{gathered}
$$


For example, if $B_{0}$ is (the completion at the irrelevant ideal of) a graded Hodge algebra on the partially ordered set $H:=\left\{X_{1}, \ldots, X_{N}\right\}$ governed by the monomial ideal $\Sigma$ (see [DEP]), ord is the associated weight function [DEP, proof of Proposition 1.1], and $\alpha\left(d, d^{\prime}\right):=d+d^{\prime}$, then $\operatorname{gr}_{F, \alpha}\left(B_{0}\right) \cong L[H] / \Sigma L[H]$ is the associated discrete Hodge algebra.

The initial ideal of $I_{0}$ with respect to the given ord and $\alpha$ is the kernel of the canonical homomorphism $\operatorname{gr}_{F, \alpha}\left(R_{0}\right) \rightarrow \mathrm{gr}_{F, \alpha}\left(B_{0}\right)$,

$$
\begin{aligned}
\operatorname{gr}_{F, \alpha}\left(I_{0}, R_{0}\right) & :=\operatorname{ker}\left(\operatorname{gr}_{F, \alpha}\left(R_{0}\right) \rightarrow \operatorname{gr}_{F, \alpha}\left(B_{0}\right)\right) \\
& \cong \bigoplus_{d \geq 0} I_{0} \cap F^{d} R_{0} / I_{0} \cap F^{d+1} R_{0} .
\end{aligned}
$$

If ord is the lexicographic order function and $\alpha$ is defined as in (24), then $\operatorname{gr}_{F, \alpha}\left(I_{0}, R_{0}\right)$ is the ideal generated in the polynomial ring $\operatorname{gr}_{F, \alpha}\left(R_{0}\right) \cong$ $L\left[X_{1}, \ldots, X_{N}\right]$ by the lexicographically first monomials of the elements of $I_{0}$.

For every power series $f \in R_{0}$ let

$$
\operatorname{ord}_{F}(f):=\sup \left\{d \in \mathbf{N} \mid f \in F^{d} R_{0}\right\} .
$$

Then from (23) we see

$$
\operatorname{ord}_{F}\left(f \cdot f^{\prime}\right) \geq \alpha\left(\operatorname{ord}_{F} f, \operatorname{ord}_{F} f^{\prime}\right) .
$$

The initial form of $f \in R_{0}$ in $\operatorname{gr}_{F, \alpha}\left(R_{0}\right)$ is defined to be

$$
\operatorname{in}_{F}(f):=\left(f \bmod F^{d+1} R_{0}\right) \in \operatorname{gr}_{F, \alpha}\left(R_{0}\right)
$$

in case $d:=\operatorname{ord}_{F} f$ is finite and is defined to be zero otherwise. A standard base of $I_{0}$ with respect to the given ord and $\alpha$ is an $n$-tuple $f=\left(f_{1}, \ldots, f_{n}\right)$ of elements from $I_{0}$ such that the initial forms $\operatorname{in}_{F}\left(f_{i}\right)$ of the coordinates of $f$ generate $\operatorname{gr}_{F, \alpha}\left(I_{0}, R_{0}\right)$. This means that

$$
I_{0} \cap F^{d} R_{0}=\left\{\sum_{i=0}^{n} r_{i} f_{i} \mid r_{i} \in R_{0}, \alpha\left(\operatorname{ord}_{F} r_{i}, \operatorname{ord}_{F} f_{i}\right) \geq d\right\}
$$

for every $d$. (" $\supseteq$ " is trivial and " $\subseteq$ " is true modulo $F^{d+1} R_{0}$, so the identity follows from the Artin-Rees lemma and the fact that $F^{k} R_{0}$ is contained for large $k$ in arbitrarily high powers of the maximal ideal.) In particular the $f_{i}$ generate $I_{0}$.

In what follows the ring $\operatorname{gr}_{F, \alpha}\left(R_{0}\right)$, which is isomorphic to $L\left[X_{1}, \ldots, X_{N}\right]$ as an $L$-vector space, will be considered to be contained in

$$
R_{0}=L\left[\left[X_{1}, \ldots, X_{N}\right]\right] \text {. }
$$

Note that then the multiplication in $\operatorname{gr}_{F, \alpha}\left(R_{0}\right)$ satisfies

$$
X^{v} \cdot X^{w}= \begin{cases}X^{v+w} & \text { if } \operatorname{ord}\left(X^{v+w}\right)=\left(\operatorname{ord}\left(X^{v}\right), \operatorname{ord}\left(X^{w}\right)\right), \\ 0 & \text { otherwise. }\end{cases}
$$


An element $f=\sum c_{v} X^{v} \in R_{0}, c_{v} \in L$, is called homogeneous of order $d$ if $c_{v} \neq 0$ implies ord $\left(X^{v}\right)=d$. Note that homogeneous elements of $R_{0}$ are automatically in $\operatorname{gr}_{F, \alpha}\left(R_{0}\right)$. Let $f_{1}, \ldots, f_{n} \in R_{0}$ be homogeneous elements. Then a homogeneous syzygy of $f:=\left(f_{1}, \ldots, f_{n}\right)$ is an $n$-tuple $r=\left(r_{1}, \ldots, r_{n}\right) \in R_{0}^{n}$ of homogeneous elements of $R_{0}$ such that $\langle r, f\rangle_{\mathrm{gr}}=0$ and $\alpha\left(\operatorname{ord}_{F} r_{i}, \operatorname{ord}_{F} f_{i}\right)$ is independent upon $i$ whenever it is defined. Here the subscript "gr" means that in the sum

$$
\langle r, f\rangle_{\mathrm{gr}}=\sum_{i=1}^{n} r_{i} f_{i}
$$

the products $r_{i} f_{i}$ should be carried out in the ring $\operatorname{gr}_{F, \alpha}\left(R_{0}\right)$. The number $\alpha\left(\operatorname{ord}_{F} r_{i}, \operatorname{ord}_{F} f_{i}\right)$ will be called the syzygy order of $r$, which must not be confused with ord $r$, the $n$-tuple of the ord $r_{i}$ 's.

(4.3) Lemma. Let $R_{0}:=L\left[\left[X_{1}, \ldots, X_{N}\right]\right]$ be a power series ring over the field $L, I_{0}$ an ideal of $R_{0}, s:=\left(s_{1}, \ldots, s_{n}\right) \in R_{0}^{n}$ a system of generators for $I_{0}$ (i.e., the coordinates of $s$ generate $I_{0}$ ), ord an order function, and $\alpha: \mathbf{N} \times$ $\mathbf{N} \rightarrow \mathbf{N}$ a commutative semigroup structure on $\mathbf{N}$ compatible with ord. Further let $r^{j}=\left(r_{1}^{j}, \ldots, r_{n}^{j}\right) \in \operatorname{gr}_{F, \alpha}\left(R_{0}\right)^{n}, j=1, \ldots, k$, be homogeneous syzygies of $\operatorname{in}_{F}(s):=\left(\operatorname{in}_{F}\left(s_{1}\right), \ldots\right.$, in $\left._{F}\left(s_{n}\right)\right)$ generating the relation module of $\operatorname{in}_{F}(s)$. Consider $\operatorname{gr}_{F, \alpha}\left(R_{0}\right) \cong L\left[X_{1}, \ldots, X_{N}\right]$ to be a ring contained in $R_{0}$ as an $L$ linear subspace. Then the following are equivalent.

(i) $s$ is a standard base of $I_{0}$.

(ii) There are $n$-tuples $h^{j}=\left(h_{1}^{j}, \ldots, h_{n}^{j}\right) \in R_{0}^{n}$ such that in $R_{0},\left\langle r^{j}, s\right\rangle=$ $\left\langle h^{j}, s\right\rangle$ for every $j$ and such that $\alpha\left(\operatorname{ord}_{F} h_{i}^{j}, \operatorname{ord}_{F} s_{i}\right)$ is greater than the syzygy order of $r^{j}$ for every $i$ (and every $j$ ).

If these conditions are satisfied, the syzygies $r^{1}-h^{1}, \ldots, r^{k}-h^{k}$ of $s$ form a generating system of the relation module of $s$.

Proof. The implication (i) $\Rightarrow$ (ii) follows directly from the fact that $I_{0} \cap F^{d} R_{0}$ coincides with

$$
I(d):=\left\{\sum r_{i} s_{i} \mid r_{i} \in R_{0}, \alpha\left(\operatorname{ord}_{F} r_{i}, \operatorname{ord}_{F} s_{i}\right) \geq d\right\}
$$

for every $d$. For, by definition of $r^{j}$,

$$
\operatorname{ord}_{F}\left\langle r^{j}, s\right\rangle>\text { syzygy order of } r^{j}, \quad \text { say } d(j),
$$

hence $\left\langle r^{j}, s\right\rangle \in I_{0} \cap F^{d(j)+1} R_{0}=I(d(j)+1)$.

Let us prove that (ii) implies (i). We have to show that $I_{0} \cap F^{d} R_{0} \subseteq I(d)$ for every $d$. Since $I_{0}=I(0)$, it suffices to prove

$$
I(k) \cap F^{d} R_{0} \subseteq I(k+1) \text { for } k<d .
$$

Let $x \in I(k) \cap F^{d} R_{0}$. Then there is some $h=\left(h_{1}, \ldots, h_{n}\right) \in R_{0}^{n}$ such that

$$
x=\langle h, s\rangle \text { and } \alpha\left(\operatorname{ord}_{F} h_{i}, \operatorname{ord}_{F} s_{i}\right) \geq k
$$


for every $i$. We may assume that equality holds in (27) for at least one $i$.

For every given $r \in R_{0}$ let $r(j), j=0,1,2, \ldots$, denote the homogeneous elements of $R_{0}$ satisfying

$$
r=\sum_{j=0}^{\infty} r(j) \quad \text { and } \quad \operatorname{ord}_{F} r(j)=j .
$$

Then, comparing coefficients in the identity $x=\langle h, s\rangle$, we obtain for the homogeneous elements of order $k$,

$$
0=\sum_{i=1}^{n}\left(h_{i} s_{i}\right)(k)
$$

i.e., there is some nonzero $n$-tuple $H=\left(H_{1}, \ldots, H_{n}\right) \in R_{0}^{n}$ such that $H_{i}$ is either zero or the initial form of $h_{i}$ and such that $H$ is a homogeneous syzygy of $\operatorname{in}_{F}(s)$ with syzygy order $k$. In particular, $H$ can be expressed in terms of the generators $r^{j}$. There are elements $a_{j} \in R_{0}$ and $h^{\prime}=\left(h_{1}^{\prime}, \ldots, h_{n}^{\prime}\right) \in R_{0}^{n}$ such that

$$
h=\sum_{j=1}^{k} a_{j} r^{j}+h^{\prime}
$$

and

$$
\begin{aligned}
& \alpha\left(\alpha\left(\operatorname{ord}_{F} a_{j} \operatorname{ord}_{F} r_{i}^{j}\right), \operatorname{ord}_{F} s_{i}\right) \geq k, \\
& \alpha\left(\operatorname{ord}_{F} h_{i}^{\prime}, \operatorname{ord}_{F} s_{i}\right) \geq k+1
\end{aligned}
$$

for every $i$. The element $x=\langle h, s\rangle$ can now be written

$$
x=\sum_{j=1}^{k}\left\langle a_{j} h^{j}, s\right\rangle+\left\langle h^{\prime}, s\right\rangle .
$$

To prove $x \in I(k+1)$, it will be sufficient to show that each term on the right is in $I(k+1)$. The second inequality of $(28)$ implies $\left\langle h^{\prime}, s\right\rangle \in I(k+1)$. Further

$$
\begin{aligned}
\alpha\left(\operatorname{ord}_{F} a_{j} h_{i}^{j}, \operatorname{ord}_{F} s_{i}\right) \\
\quad \geq \alpha\left(\alpha\left(\operatorname{ord}_{F} a_{j} \operatorname{ord}_{F} h_{i}^{j}\right), \operatorname{ord}_{F} s_{i}\right) \\
\quad=\alpha\left(\operatorname{ord}_{F} a_{j}, \alpha\left(\operatorname{ord}_{F} h_{i}^{j}, \operatorname{ord}_{F} s_{i}\right)\right) \\
\left.\quad \geq \alpha\left(\operatorname{ord}_{F} a_{j}, d(j)\right)+1 \quad \text { by hypothesis }(\mathrm{ii})\right) \\
\quad=\alpha\left(\operatorname{ord}_{F} a_{j}, \min _{i} \alpha\left(\operatorname{ord}_{F} r_{i}^{j}, \operatorname{ord}_{F} s_{i}\right)\right)+1 \\
\quad=\min \alpha\left(\operatorname{ord}_{F} a_{j}, \alpha\left(\operatorname{ord}_{F} r_{i}^{j}, \operatorname{ord}_{F} s_{i}\right)\right)+1 \\
\quad \geq k+1 \quad(\operatorname{by}(28)) .
\end{aligned}
$$

Therefore $\left\langle a_{j} h^{j}, s\right\rangle \in I(k+1)$ as required. This proves (26), and hence that (ii) implies (i). 
We have yet to prove that $r^{1}-h^{1}, \ldots, r^{k}-h^{k}$ generate the relation module $Z(s)$ of $s$. Let $h=\left(h_{1}, \ldots, h_{n}\right) \in R_{0}^{n}$ be a syzygy of $s$ such that

$$
\alpha\left(\operatorname{ord}_{F} h_{i}, \operatorname{ord}_{F} s_{i}\right) \geq q
$$

for every $i$. Then, using the same arguments as above, we see that there are elements $a_{j} \in R_{0}$ and $h^{\prime}=\left(h_{1}^{\prime}, \ldots, h_{n}^{\prime}\right) \in R_{0}^{n}$ such that

$$
h=\sum_{j=1}^{k} a_{j}\left(r^{j}-h^{j}\right)+h^{\prime}
$$

and

$$
\alpha\left(\operatorname{ord}_{F} h_{i}^{\prime}, \operatorname{ord}_{F} s_{i}\right) \geq q+1 .
$$

Iterating identity (29), we see that every syzygy $h$ of $s$ can be written

$$
h \equiv \sum_{j=1}^{k} a_{j}\left(r^{j}-h^{j}\right) \quad \bmod Z(s) \cap\left(F^{q} R_{0}\right)^{n}
$$

with suitable coefficients $a_{j} \in R_{0}$ and $q$ arbitrarily large. In particular, $q$ can be chosen such that $F^{q} R_{0}$ is contained in arbitrarily high powers of the maximal ideal $\mathfrak{M}_{0}$ of $R_{0}$, i.e., by the Artin-Rees lemma,

$$
Z(s)=\sum_{j=1}^{k} R_{0}\left(r^{j}-h^{j}\right)+\mathfrak{M}_{0} \cdot Z(s) .
$$

Hence the syzygies $r^{j}-h^{j}$ generate $Z(s)$. Q.E.D.

(4.4) Definition. Let $S$ be a locally Noetherian scheme, $E$ a locally free sheaf on $S$ of finite rank $r$, and

$$
V:=\mathbf{V}(E)=\operatorname{Spec} \mathbf{S}(E)
$$

the associated vector bundle on $S$. A family of affine cones over $S$ is a closed subscheme

$$
X:=\operatorname{Spec} \mathbf{S}(E) / I \hookrightarrow V
$$

defined by a sheaf $I$ of homogeneous ideals of the symmetric algebra $\mathbf{S}(E)$ of $E$ over $\mathscr{O}_{S}$ such that the composition $X \rightarrow V \rightarrow S$ is a flat morphism.

(4.5) Proposition. Let $X \rightarrow V \rightarrow S$ be a family of affine cones over a locally Noetherian scheme $S$, and let $s_{0} \in S$ be a point such that the normal sheaf of the fiber $X_{s_{0}}$ over $s_{0}$ in $V_{s_{0}}$ has no nonzero homogeneous sections of degree less than minus one,

$$
\mathscr{N}_{X_{s_{0}} / V_{s_{0}}}(<-1)=0 \text {. }
$$

Then the same is true for all points $s$ from a neighborhood $U$ of $s_{0}$,

$$
\mathscr{N}_{X_{s} / V_{s}}(<-1)=0 \text { for } s \in U \text {. }
$$


Proof. The assertion is local. So we may assume

$$
S=\operatorname{Spec} A, \quad V=\operatorname{Spec} A[T]=\mathbf{A}_{S}^{N}, \quad X=\operatorname{Spec} A[T] / I_{A} .
$$

Here $A$ is a Noetherian ring, $T=\left\{T_{1}, \ldots, T_{N}\right\}$ is a finite set of indeterminates, and $I_{A}$ is a homogeneous ideal of the graded ring

$$
R_{A}:=A[T]=\bigoplus_{d=0}^{\infty} R_{A}(d), \quad R_{A}(d):=\bigoplus_{\sum a_{i}=d} A T_{1}^{a_{1}} \cdots T_{N}^{a_{N}}
$$

such that $B_{A}:=R_{A} / I_{A}$ is flat over $A$. For every $s \in \operatorname{Spec} A$ let

$$
B_{k(s)}:=B_{A} \otimes_{A} k(s), \quad R_{k(s)}:=R_{A} \otimes_{A} k(s),
$$

where $k(s)$ is the residue class field of $s$, and let $I_{k(s)}$ denote the kernel of the canonical homomorphism $R_{k(s)} \rightarrow B_{k(s)}$. Then the normal sheaf of $X_{s}$ in $V_{s}$ is the $\mathscr{O}_{X_{s}}$-module associated with

$$
N_{I_{k(s)}}:=\operatorname{Hom}_{R_{k(s)}}\left(I_{k(s)}, B_{k(s)}\right) .
$$

Consider a complex $L: \cdots \rightarrow L^{p-1} \rightarrow L^{p} \rightarrow L^{p+1} \rightarrow \cdots$ of finitely generated flat modules over $A$ and the additive functors

$$
T^{i}: A \text {-Mod } \rightarrow A \text {-Mod, } \quad M \mapsto H^{i}\left(L \otimes_{A} M\right),
$$

associated with $L$. Then it is well known (and easy to see, cf. [Ha, Theorem 12.8]) that the functions

$$
\operatorname{Spec} A \rightarrow \mathbf{N}, \quad p \mapsto h^{i}(p, L):=\operatorname{dim}_{k(p)} T^{i}(k(p))
$$

are upper semicontinuous with respect to the Zariski topology on $\operatorname{Spec} A$.

The assertion of the proposition will be proved if we can show that there is some complex $L$ as above such that

$$
N_{I_{k(s)}}(<-1) \cong T^{0}(k(s))
$$

for every $s \in \operatorname{Spec} A$. Let

$$
\cdots \rightarrow R_{A}^{r_{1}} \rightarrow R_{A}^{r_{0}} \rightarrow I_{A} \rightarrow 0
$$

be a graded free resolution of $I_{A}$ over $R_{A}$. Apply the functor $\operatorname{Hom}_{R_{A}}\left(\square, B_{A}\right)$ to get a complex of free $B_{A}$-modules

$$
0 \rightarrow B_{A}^{r_{0}} \rightarrow B_{A}^{r_{1}} \rightarrow \cdots
$$

and take the subcomplex over $A$ generated by the homogeneous elements of degree less than minus one:

$$
L: 0 \rightarrow\left(B_{A}^{r_{0}}\right)(<-1) \rightarrow\left(B_{A}^{r_{1}}\right)(<-1) \rightarrow \cdots .
$$

We shall show this is the complex we are looking for. Apply the functor $\otimes_{A} k(s)$ to the complex (30). The resulting complex

$$
\cdots \rightarrow R_{k(s)}^{r_{1}} \rightarrow R_{k(s)}^{r_{0}} \rightarrow I_{A} \otimes_{A} k(s) \rightarrow 0
$$


is a graded free resolution of $I_{A} \otimes_{A} k(s)$ over $R_{k(s)}$, since its $i$ th homology is $H_{i}=\operatorname{Tor}_{i}^{A}\left(I_{A}, k(s)\right)=0$. Note that $I_{A}$ is $A$-flat, since $R_{A}$ and $B_{A}$ have this property. From the definition of $I_{k(s)}$ and the fact that $B_{A}$ is $A$-flat, we see that $I_{k(s)} \cong I_{A} \otimes_{A} k(s)$, so (32) may be considered as a graded free resolution of $I_{k(s)}$ over $R_{k(s)}$. Apply $\operatorname{Hom}_{R_{k(s)}}\left(\square, B_{k(s)}\right)$ to obtain an exact sequence

$$
0 \rightarrow N_{k(s)} \rightarrow B_{k(s)}^{r_{0}} \rightarrow B_{k(s)}^{r_{1}} \text {. }
$$

All the homomorphisms above between free modules over $R_{A}, B_{A}, R_{k(s)}$, $B_{k(s)}$ can be represented by matrices with elements from $R_{A}$. Application of the functor $\otimes_{A} k(s)$ does not change the matrix and application of the contravariant Hom-functors replaces the matrices by its transposes. Taking this into account, it is easily seen that the second map of (33) is given by the same matrix like the first map of (31). So application of $\otimes_{A} k(s)$ to (31) gives the exact sequence (33). Taking elements of degree less than minus one, we obtain the exact sequence

$$
0 \rightarrow N_{I_{k(s)}}(<-1) \rightarrow L^{0} \otimes_{A} k(s) \rightarrow L^{1} \otimes_{A} k(s) .
$$

This proves

$$
N_{I_{k(s)}}(<-1) \cong H^{0}\left(L \otimes_{A} k(s)\right)=T^{0}(k(s)) \text {. Q.E.D. }
$$

The following assertion can be considered, in some sense, as a special case of (4.5), but seems to be more important for applications. We give an independent proof.

(4.6) Proposition. Let $R_{0}:=L\left[\left[X_{1}, \ldots, X_{N}\right]\right]$ and let $I_{0}$ be a homogeneous ideal of $R_{0}$ (with respect to the usual grading by total degree). Further let ord be an order function on the power products in $X_{1}, \ldots, X_{N}$ and $\alpha$ a commutative group structure on $\mathbf{Z}$ compatible with the natural order on $\mathbf{Z}$ and such that

$$
\alpha\left(\operatorname{ord}\left(X^{v}\right), \operatorname{ord}\left(X^{v^{\prime}}\right)\right)=\operatorname{ord}\left(X^{v+v^{\prime}}\right) .
$$

Consider $\operatorname{gr}_{F, \alpha}\left(R_{0}\right)$ as an L-linear subspace of $R_{0}$.

If $B_{0}^{\prime}:=R_{0} / I_{0}^{\prime}$ has only tangentially flat deformations with $I_{0}^{\prime}$ the ideal generated by the initial forms $\operatorname{in}_{F}(f) \in \mathrm{gr}_{F, \alpha}\left(R_{0}\right)$ of the elements $f \in I_{0}$, the same is true for $B_{0}:=R_{0} / I_{0}$.

The following implication is true:

$$
N_{\mathrm{gr}_{F, \alpha}\left(I_{0}, R_{0}\right)}(<-1)=0 \Rightarrow N_{\mathrm{gr}\left(I_{0}, R_{0}\right)}(<-1)=0,
$$

when $\operatorname{gr}_{F, \alpha}\left(R_{0}\right)$ is considered to be $\mathbf{N}$-graded with the graded structure coming from the usual graded structure of $L\left[X_{1}, \ldots, X_{N}\right]$ defined by total degree.

The proof follows from the lemma below, which uses the notion of graded structure in a slightly generalized sense. We say a module $N$ over an $\mathbf{N}$-graded ring $R=\bigoplus R(d)$ has a generalized graded structure over $R$ if

$$
\bigoplus_{d \in \mathbf{Z}} N(d) \subseteq N \subseteq \prod_{d \in \mathbf{Z}} N(d)
$$


for suitable $R(0)$-submodules $N(d)$ of $N$ satisfying $R\left(d^{\prime}\right) \cdot N(d) \subseteq N\left(d+d^{\prime}\right)$ and called submodules of homogeneous elements of degree $d$. The notion of graded homomorphism (of degree zero) is defined in the usual way.

(4.7) Lemma. Let $R$ be a Noetherian ring equipped with a descending filtration

$$
F R: \cdots \supseteq F^{d} R \supseteq F^{d+1} R \supseteq \cdots \quad(d \in \mathbf{N})
$$

by ideals $F^{d} R$ and $\alpha$ a commutative group structure on $\mathbf{Z}$, which is compatible with the natural order on $\mathbf{Z}$, has identity element $0 \in \mathbf{N}$, and is such that $F^{d} R \cdot F^{d^{\prime}} R \subseteq F^{\alpha\left(d, d^{\prime}\right)} R$. Then

$$
\operatorname{gr}_{F, \alpha}(R):=\bigoplus_{d \in \mathbf{N}} F^{d} R / F^{d+1} R
$$

admits canonically the structure of an $(\mathbf{Z}, \alpha)$-graded ring such that

$$
\operatorname{gr}_{F, \alpha}(I, R):=\bigoplus_{d \in \mathrm{N}} I \cap F^{d} R+F^{d+1} R / F^{d+1} R
$$

is a homogeneous ideal. So $\mathrm{gr} N_{I}$ and $N_{\mathrm{gr}_{F, \alpha}(I, R)}$ are well defined $(\mathbf{Z}, \alpha)$-graded modules (the latter in the generalized sense).

There is a graded injective homomorphism of degree zero,

$$
\operatorname{gr} N_{I} \rightarrow N_{\mathrm{gr}_{F, \alpha}(I, R)} .
$$

Moreover, if $R=\bigoplus_{k \in \mathbf{N}} R(k)$ is an $\mathbf{N}$-graded ring, $I=\bigoplus_{k \in \mathbf{N}} I(k)$ a homogeneous ideal, and the filtration $F R$ of $R$ is compatible with the graded structure,

$$
F^{d} R=\bigoplus_{k \in N} F^{d} R(k), \quad F^{d} R(k):=F^{d} R \cap R(k),
$$

then the $\mathbf{N}$-graded structure on $R$ induces $\mathbf{Z}$-graded structures on $\operatorname{gr}_{F, \alpha}(R)$, $\operatorname{gr}_{F, \alpha}(I, R), \operatorname{gr} N_{I}$, and $N_{\mathrm{gr}_{F, \alpha}(I, R)}$ (for the latter in the generalized sense) such that (35) is also graded of degree zero with respect to these additional graded structures. In particular, if $\mathrm{gr}_{F, \alpha}(R)$ is Noetherian, $N_{\mathrm{gr}_{F, \alpha}(I, R)}$ is considered to be $\mathbf{Z}$-graded with respect to the graded structure coming from $R$, and if

$$
N_{\mathrm{gr}_{F, \alpha}(I, R)}(<-1)=0
$$

and the filtration induced by $F R$ on $R / I$ is separated, then $N_{I}(<-1)=0$. Proof. We shall write " $i+j$ " instead of $\alpha(i, j)$. To construct the homomorphism (35), let $G \in \operatorname{gr} N_{I}$ be some homogeneous element of degree $d$ and take any

$$
g \in N_{I}=\operatorname{Hom}_{R}(I, R / I)
$$

with initial form $\operatorname{in}(g):=\left(g \bmod F_{N_{I}}^{d+1}\right)=G$. Then

$$
g\left(I \cap F^{j} R\right) \subseteq F^{j+d} R+I / I
$$


for every $j$. Hence $g$ induces homomorphisms

$$
g(j): I \cap F^{j} R / I \cap F^{j+1} R \rightarrow F^{j+d} R+I / F^{j+d+1} R+I
$$

defining a homogeneous element of degree $d$ of $N_{\mathrm{gr}_{F, \alpha}(I, R)}$. If $g^{\prime} \in N_{I}$ is another element with initial form $G$, then $g-g^{\prime} \in F_{N_{l}}^{d+1}$, hence $g(j)=g^{\prime}(j)$ for every $j$. This proves there is a well-defined graded homomorphism of degree zero,

$$
\operatorname{gr} N_{I} \rightarrow N_{\mathrm{Br}_{F, \alpha}(I, R)}, \quad G=\operatorname{in}(g) \mapsto \bigoplus_{j \in \mathbf{Z}} g(j) .
$$

Suppose $g(j)=0$ for every $j$. Then

$$
g\left(I \cap F^{j} R\right) \subseteq F^{j+d+1} R+I / I,
$$

hence $G=\left(g \bmod F_{N_{I}}^{d+1}\right)=0$. The homomorphism is injective.

Assume $R$ is $\mathbf{N}$-graded, $I$ is homogeneous, and the filtration is compatible with the graded structure. Then the induced Z-graded structures on $\operatorname{gr}_{F, \alpha}(R)$, $\operatorname{gr}_{F, \alpha}(I, R), \operatorname{gr} N_{I}, N_{\mathrm{gr}_{F, \alpha}(I, R)}$ are given, respectively, by

$$
\begin{aligned}
& \operatorname{gr}_{F, \alpha}(R)(k)=\bigoplus_{d \in \mathbf{N}} F^{d} R(k) / F^{d+1} R(k), \\
& \operatorname{gr}_{F, \alpha}(I, R)(k)=\bigoplus_{d \in \mathbf{N}} I(k) \cap F^{d} R(k)+F^{d+1} R(k) / F^{d+1} R(k), \\
& \left(\operatorname{gr} N_{I}\right)(k)=\left\{\begin{array}{l|l}
\left(g_{d} \bmod F_{N_{I}}^{d+1}\right)_{d \in \mathbf{Z}} & \begin{array}{c}
g_{d}(I(j)) \subseteq R(j+k) / I(j+k) \\
\text { for every } j
\end{array}
\end{array},\right. \\
& \left(N_{\mathrm{gr}_{F, \alpha}(I, R)}\right)(k)=\left\{\begin{array}{l|l}
g \in N_{\mathrm{gr}_{F, \alpha}(I, R)} & g\left(\operatorname{gr}_{F, \alpha}(I, R)(j)\right) \subseteq \frac{\mathrm{gr}_{F, \alpha}(R)(j+k)}{\operatorname{gr}_{F, \alpha}(I, R)(j+k)} \\
\text { for every } j
\end{array}\right\} .
\end{aligned}
$$

Therefore, if $G:=\left(g_{d} \bmod F_{N_{I}}^{d+1}\right)_{d \in \mathbf{Z}}$ is in $\left(\operatorname{gr} N_{I}\right)(k)$,

$$
\begin{aligned}
& g_{i}\left(I(j) \cap F^{d} R(j)\right) \subseteq \frac{R(j+k)}{I(j+k)} \cap \frac{F^{d+1} R+1}{I} \\
&=\frac{F^{d+i} R(j+k)+I(j+k)}{I(j+k)},
\end{aligned}
$$

so that the image of $G$ in $N_{\mathrm{gr}_{F, \alpha}(I, R)}$ is homogeneous of degree $k$. In other words, the homomorphism (35) respects the graded structures coming from that of $R$.

Finally assume $N_{\mathrm{gr}_{F, \alpha}(I, R)}(<-1)=0$ and let $g \in N_{I}$ be homogeneous of degree $k<-1$ and of $F_{N_{I}}$-order $d$. Then

$$
\operatorname{in}(g)=\left(g \bmod F_{N_{l}}^{d+1}\right) \in \operatorname{gr} N_{I}
$$


gives a homogeneous element of degree $k$ of $N_{\mathrm{gr}_{F, \alpha}(I, R)}$, so in $(g)$ must be zero, i.e., $g$ has infinite $F_{N_{I}}$-order. If the filtration induced by $F R$ on $R / I$ is separated, this implies $g=0$. Q.E.D.

(4.8) Example. Let $B_{0}$ be the local ring at the vertex of the affine cone over the Grassmann variety $G_{d, n}$ of $d$-planes in $n$-space (with respect to the usual embedding by Grassmann coordinates). Then the ring $B_{0}$ has only tangentially flat deformations in case $(d, n) \neq(2,4)$ and is a complete intersection otherwise.

Proof. The graded ring $\operatorname{gr}\left(B_{0}\right)$ is just the homogeneous coordinate ring of $G_{d, n}$, hence admits an ordinal Hodge algebra structure on the poset $H=\left\{\begin{array}{l}n \\ d\end{array}\right\}$ consisting of the symbols

$$
X_{[i .]} \quad\left([i .]=\left[i_{1}, \ldots, i_{d}\right], \quad 1 \leq i_{1}<\cdots<i_{d} \leq n\right)
$$

and equipped with the partial order

$$
[i .] \leq\left[i^{\prime} .\right] \text { if } i_{k} \leq i_{k}^{\prime} \text { for } k=1, \ldots, d
$$

(see [DEP, Theorem 11.1]). The associated discrete Hodge algebra is

$$
B_{00}:=L[H] / \Sigma L[H]
$$

with $L$ the residue class field of $B_{0}$ and $\Sigma$ the ideal of monomials generated by the products $X_{[i .]} \cdot X_{[j .]}$ with $[i$.$] and [j$.$] incomparable. In case n=4$, $d=2$ there is only one such product, $X_{[1,4]} \cdot X_{[2,3]}$. So $B_{00}$ is a complete intersection and hence $B_{0}$ is.

Returning to the general case, we want to show $B_{0}$ has only tangentially flat deformations. For this we shall apply Proposition (4.6) with "ord" the weight function on the monimials in the variables from $H$ defined by the partial order of $H$ (see [DEP, proof of Proposition 1.1]). We see that it is sufficient to show

$$
N_{B_{00}}(<-1)=0
$$

when $N_{B_{00}}$ is considered to have the graded structure coming from the graded structure of the polynomial ring $L[H]$ defined by total degree. Let $S$ be the set of generators $X_{[i .]} \cdot X_{[j .]}$ of $\Sigma$. By Proposition (3.6) it suffices to show that every 1-component of $S$ consists of at least two elements. In what follows we shall say that a finite ascending sequence of integers $x_{1}<x_{2}<\cdots<x_{k}$ has a gap between $x_{i}$ and $x_{i+1}$ if $x_{i+1}-x_{i}>1$, and the number

$$
g:=\sum_{i=2}^{k}\left(x_{i}-x_{i-1}-1\right)=x_{k}-x_{1}-(k-1)
$$

will be called the gap number of the sequence.

Assume there is a 1-component of $S$ containing only one element, say $X_{[i .]}$. $X_{[j .]}$. Then, since $[i$.$] and [j$.$] are incomparable, we may assume that$

$$
(1 \leq) i_{a}<j_{a}<\cdots<j_{k}<\cdots<j_{b}<i_{b}(\leq n)
$$


for suitable subscripts $a, b$. In particular $d \geq b-a+1$ and $n \geq b-a+3$. There cannot exist a variable different from $X_{\left[i_{0}\right]}$ and $X_{[j .]}$ and incomparable with one of these two variables, since otherwise the 1-component of $X_{\left[i_{0}\right]} \cdot X_{[j .]}$ would have another element. In case the sequence (36) has one or more gaps, such a variable exists, i.e, this cannot happen. From (36) we see that each of the sequences $0<j_{1}<\cdots<j_{a}$ and $j_{b}<\cdots<j_{d}<n+1$ has at least one gap, so that there is more than one possible choice for the integers $j_{k} \notin\left\{j_{a}, j_{a+1}, \ldots, j_{b}\right\}$ provided there are such integers $j_{k}$. Hence there are not, i.e., $d=b-a+1$, $a=1, b=d$.

By (36), the sequence $i_{1}<\cdots<i_{k}<\cdots<i_{d}$ has gap number equal to two. So there cannot be any $i_{k}$ strictly between $i_{1}$ and $i_{d}$ (there would be several choices for $i_{k}$ ), i.e., $d=2$.

The gap number of $0<i_{1}<i_{2}<n+1$ is $(n+1)-3=n-2$. So in case $n \geq 5$ there is a gap between 0 and $i_{1}$ or $i_{2}$ and $n+1$, which is impossible. Therefore, $n=4$ and $d=2$. But this situation has already been treated. Q.E.D.

(4.9) Example. Let $\Omega_{a_{1}, \ldots, a_{d}}^{n}$ be the Schubert variety of $d$-planes $W$ in $n$ space $V$ intersecting a given flag $0 \varsubsetneqq V_{1} \varsubsetneqq \cdots \varsubsetneqq V_{n}=V$ such that

$$
\operatorname{dim}\left(W \cap V_{n-d+k-a_{k}}\right) \geq k \text { for } k=1, \ldots, d .
$$

Then the local ring $B_{0}$ at the vertex of the affine cone over $\Omega_{a_{1}, \ldots, a_{d}}^{n}$ with respect to the embedding by Grassmann coordinates has only tangentially flat deformations in the case $\Omega_{a_{1}, \ldots, a_{d}}^{n} \not \neq G_{2,4}, \Omega_{1,0}^{4}$ and is a complete intersection otherwise.

Proof. We may assume that at least one $a_{k}$ is positive, the case $a_{k} \leq 0$ for all $k$ being treated in the previous example. The graded ring $\operatorname{gr}\left(B_{0}\right)$ is obtained from the coordinate ring of $G_{d, n}$ as a factor ring modulo the ideal generated by the indeterminates $X_{\left[i_{1}, \ldots, i_{d}\right]}$ such that

$$
i_{k} \leq a_{d-k+1}+(k-1) \text { for } k=1, \ldots, d
$$

(skip the inequalities with nonpositive $\left.a_{d-k+1}\right)$. Therefore, $\operatorname{gr}\left(B_{0}\right)$ admits the structure of an ordinal Hodge algebra on the poset

$$
H^{\prime}:=\left\{\begin{array}{l|l}
X_{[i .]} \in H & \begin{array}{l}
i_{k} \nless a_{d-k+1}+(k-1) \text { for at least } \\
\text { one } k \text { such that } a_{d-k+1} \text { is positive }
\end{array}
\end{array}\right\}
$$

with the partial order induced from the partial order of the poset $H$ of the previous example (see [DEP, Proposition 1.2 and $\S 11$ ]). Let $S^{\prime}$ be the set of generators for the associated ordinal ideal of monomials. As in the previous example it will be sufficient to show the 1-components of $S^{\prime}$ contain at least two elements. Suppose there is a generator

$$
X_{[i .]} \cdot X_{[j .]} \in S^{\prime}
$$


whose 1-component contains no further element. Then there is no variable in $H^{\prime}$ different from $X_{[i .]}$ and $X_{[j .]}$ and incomparable with one of these variables. As in the previous example, we may assume

$$
(1 \leq) i_{a}<j_{a}<\cdots<j_{k}<\cdots<j_{b}<i_{b}(\leq n)
$$

for suitable subscripts $a, b$. If there is some $i_{k}$ or $j_{k}$ that can be increased preserving these inequalities, we get a contradiction. So the following sequences have no gap:

$$
\begin{array}{r}
i_{1}<\cdots<i_{a}, i_{a+1}<\cdots<i_{b}<i_{b+1}<\cdots<i_{d}<n+1, \\
\wedge \\
j_{1}<\cdots<j_{a}<j_{a+1}<\cdots<j_{b}, j_{b+1}<\cdots<j_{d}<n+1 .
\end{array}
$$

The gap numbers of $i_{a}<i_{a+1}$ and $j_{b}<j_{b+1}$ are 2 and 1, respectively. In particular,

$$
n=i_{1}+d+1=j_{1}+d, \quad i_{1}<j_{1},
$$

and

$$
\begin{array}{ll}
i_{k}<j_{k} & \text { for } k=1, \ldots, a, \\
j_{k}<i_{k} & \text { for } k=a+1, \ldots, b .
\end{array}
$$

So, if $a \neq 1$, one could increase $i_{a}$. Hence $a=1$. Similarly, if $a+1 \neq b$, one could increase $j_{b}$. Hence $b=2$.

If $i_{1}>1, X_{\left[i_{1}-1, i_{2}, \ldots, i_{d}\right]} \notin H^{\prime}$, hence $i_{1}-1=a_{d-1+1}+(1-1)$, i.e, $a_{d}=$ $i_{1}-1 \geq 1$. Therefore $\operatorname{dim} W \cap V_{n-d+d-a_{d}} \geq d$ implies

$$
W \subseteq V_{n-1} \text {. }
$$

Note that $W \cap V_{n-d+k-a_{k}}=W \cap V_{(n-1)-d+k-\left(a_{k}-1\right)}$. Therefore, the substitutions $i_{k} \mapsto i_{k}-1, a_{k} \mapsto a_{k}-1, n \mapsto n-1$ define an isomorphism

$$
\Omega_{a_{1}, \ldots, a_{d}}^{n} \cong \Omega_{a_{1}-1, \ldots, a_{d}-1}^{n-1} .
$$

This shows that we may assume $i_{1}=1$ and hence $n=d+2$.

If $b \neq d, X_{\left[j_{1}, \ldots, j_{b+1}-1, \ldots, j_{d}\right]} \notin H^{\prime}$, hence $j_{b+1}-1=a_{d-b-1+1}+b$. Note that $b=2, j_{b+1}=5$. Therefore $a_{d-2}=2$. This corresponds to the condition

$$
W \supseteq V_{d-2} \text {. }
$$

The other conditions, $k \leq \operatorname{dim} W \cap V_{n-d+k-a_{k}}=\operatorname{dim} W \cap V_{k+2-a_{k}}$, give a contradiction if one $a_{k}$ is greater than 2 , and are otherwise empty for $k=$ $1, \ldots, d-3$. Therefore,

$$
\Omega_{a_{1}, \ldots, a_{d}}^{n} \cong \begin{cases}\varnothing & \text { if some } a_{k}>2, \\ \Omega_{a_{d-1}-(d-2), a_{d}-(d-2)}^{n-(d-2)} & \text { otherwise. }\end{cases}
$$

So we are reduced to the case $n=4, d=2$. 
In case $a_{2}=a_{d} \geq 1$ we see as above $\Omega_{a_{1}, a_{2}}^{4} \cong \Omega_{a_{1}-1, a_{2}-1}^{3}$, which we already know to be impossible. So we may assume $a_{2}=0$, i.e.,

$$
\Omega=\Omega_{a_{1}, 0}^{4}=\left\{W \subseteq V \mid \operatorname{dim} W \cap V_{3-a_{1}} \geq 1\right\}
$$

$(\operatorname{dim} V=4, \operatorname{dim} W=2)$. Then $(0<) \quad a_{1} \leq 2$, since otherwise $\Omega=\varnothing$. In case $a_{1}=2, \Omega=\mathbf{P}^{2}=\Omega_{0}^{3}$, which we excluded from the beginning. In the remaining case $a_{1}=1, \Omega=\Omega_{1,0}^{4}$, the cone over $\Omega$ is easily seen to be a complete intersection. Q.E.D.

(4.10) Example. Let $0<d_{1}<\cdots<d_{e}<n$ be integers and let $F^{n}\left(d_{1}, \ldots, d_{e}\right)$ denote the variety of flags $V_{d_{1}} \varsubsetneqq \cdots \varsubsetneqq V_{d_{e}}$ in $n$-space $V$ satisfying $\operatorname{dim} V_{d_{k}}=d_{k}$ for $k=1, \ldots, e$. Consider $F^{n}\left(d_{1}, \ldots, d_{e}\right)$ to be embedded in the usual way by Grassmann coordinates:

$$
F^{n}\left(d_{1}, \ldots, d_{e}\right) \hookrightarrow \prod_{k=1}^{e} G_{d_{k}, n} \hookrightarrow \prod_{k=1}^{e} \mathbf{P}\left(\bigwedge^{d_{k}} V\right)
$$

Then the local ring $B_{0}$ at the vertex of the affine cone over $F^{n}\left(d_{1}, \ldots, d_{e}\right)$ in $\prod_{k=1}^{e} \wedge^{d_{k}} V$ has only tangentially flat deformations in case $\left(n, e, d_{e}\right) \neq$ $(4,1,2)$ and is a complete intersection otherwise.

Proof. The graded ring $\operatorname{gr}\left(B_{0}\right)$ admits an ordinal Hodge algebra structure on the poset $H^{\prime}:=\bigcup_{k=1}^{e}\left\{\begin{array}{c}h \\ d_{k}\end{array}\right\}$ of symbols $X_{[i .]}$ such that $[i]=.\left[i_{1}, \ldots, i_{d_{k}}\right], 1 \leq i_{1}<$ $\cdots<i_{d_{k}} \leq n, k=1, \ldots, e$, with the partial order defined by $\left[i_{1}, \ldots, i_{d_{k}}\right] \leq$ $\left[j_{1}, \ldots, j_{d_{k^{\prime}}}\right]$ if $d_{k} \geq d_{k^{\prime}}$ and $i_{1} \leq j_{1}, \ldots, i_{d_{k^{\prime}}} \leq j_{d_{k^{\prime}}}$ (see [DEP, §17]). Let $S^{\prime}$ be the set of products $X_{[i .]} \cdot X_{[j .]}$ with $[i]$ and $[j$.] incomparable. As above it will be sufficient to show that the 1-components of $S^{\prime}$ contain at least two elements.

Let the product $X_{[i .]} \cdot X_{[j .]}$ be in $S^{\prime},[i]=.\left[i_{1}, \ldots, i_{d_{k}}\right]$ and $[j]=.\left[j_{1}, \ldots\right.$, $j_{k^{\prime}}$ ] are incomparable. We have to find an element of $H^{\prime}$ different from the variables $X_{[i .]}$ and $X_{\left[j_{0}\right]}$ and incomparable with at least one of them. Assume $d_{k} \geq d_{k^{\prime}}$ (hence $k \geq k^{\prime}$ ). In case this inequality is strict, such a new variable is

$$
X_{\left[i^{\prime}\right]}, \quad\left[i_{.}^{\prime}\right]:=\left[i_{1}, \ldots, i_{d_{k^{\prime}}}\right],
$$

with $\left[i\right.$.] obtained from [i.] by omitting coordinates. So assume $k=k^{\prime}$. But then we are in a situation already considered. From Example (4.8) we know there is a variable of the kind we are looking for, except in the case $n=4$, $d_{k}=2$. In this latter case we may assume,

$$
[i .]=[1,4], \quad[j .]=[2,3] .
$$

If $d_{k} \neq d_{e}$ (i.e., $d_{e}=3$ ), the variable $X_{\left[j^{\prime}\right]}$ with $\left[j^{\prime}\right]=[2,3,4]$ is in $H^{\prime}$ and incomparable with $X_{[i .]}$. So we are reduced to the case $\left(n, d_{e}\right)=(4,2)$. The 
case $e=1$ has already been treated in Example (4.8), since $F^{4}(2)=G_{2,4}$. So the only remaining case is that of the flat variety $F^{4}(1,2)$. But then $X_{[1]}$ is in $H^{\prime}$ and is incomparable with $X_{[j .]}=X_{[2,3]}$. Q.E.D.

(4.11) Remark. In the same style as in the above examples one can treat determinantal varieties, Pfaffian varieties, varieties of complexes, and many others. We shall give here only one further example, the Veronese varieties.

(4.12) Example. Let $V_{m, n}$ denote the Veronese variety, i.e., the image of the $m$-tuple embedding $\mathbf{P}^{n} \rightarrow \mathbf{P}^{N}, N:=\left(\begin{array}{c}m+n \\ n\end{array}\right)-1$. Then the local ring $B_{0}$ at the vertex of the affine cone over $V_{m, n}$ has only tangentially flat deformations in case $(m, n) \neq(2,1)$ and is a complete intersection otherwise.

Proof. Let $X:=\left\{X_{a}\right\}$ be a set of $\left(\begin{array}{c}m+n \\ n\end{array}\right)$ indeterminates $X_{a}=X_{a(0), \ldots, a(n)}$ such that $|a|:=\sum_{i=0}^{n} a(i)$ equals $m$ and the coordinates of $a=(a(0), \ldots, a(n))$ are nonnegative integers. Fix a second set of indeterminates, $Y=\left\{Y_{0}, \ldots, Y_{n}\right\}$, and consider the homomorphism of polynomial algebras over $L$,

$$
f: L[X] \rightarrow L[Y], \quad X_{a}=X_{a(0), \ldots, a(n)} \mapsto Y^{a}:=Y_{0}^{a(0)} \cdots Y_{n}^{a(n)},
$$

with $L$ the residue class field of $B_{0}$. Then

$$
\operatorname{gr}\left(B_{0}\right) \cong L[X] / \operatorname{ker} f
$$

is the homogeneous coordinate ring of $V_{m, n}$.

As in the above examples the proof is based on the fact that $\operatorname{gr}\left(B_{0}\right)$ admits a certain Hodge algebra structure over $L$, which we are going to describe now. Equip the power products $\left\{Y^{v} \mid v \in \mathbf{N}^{n+1}\right\}$ with the lexicographic order,

$$
\begin{aligned}
Y^{v} & \leq Y^{v^{\prime}} \text { if }|v|<\left|v^{\prime}\right| \text { or }|v|=\left|v^{\prime}\right| \text { and the first } \\
& \text { nonzero coordinate of } v-v^{\prime} \text { is positive. }
\end{aligned}
$$

Given two variables $X_{a}, X_{b} \in X$, write $X_{a}<X_{b}$ if $Y^{a}=f\left(X_{a}\right)$ precedes $Y^{b}=$ $f\left(X_{b}\right)$ lexicographically. Identify the power products of the variables $X_{a} \in X$ with the set $\mathbf{N}^{X}$ and extend the linear order " $<$ " on $X$ to the corresponding lexicographic order on $\mathbf{N}^{X}$. We shall say the two power products $M, M^{\prime} \in \mathbf{N}^{X}$ are equivalent, symbolically $M \sim M^{\prime}$, if they have the same image under $f$. Note that

$$
X_{a_{1}}^{v(1)} \cdots X_{a_{k}}^{v(k)} \sim X_{b_{1}}^{w(1)} \cdots X_{b_{l}}^{w(l)} \quad \text { iff } \quad \sum_{i=1}^{k} v(i) a_{i}=\sum_{j=1}^{l} w(j) b_{j} .
$$

This is obviously an equivalence relation. A power product $M \in \mathbf{N}^{X}$ is defined to be a standard monomial if it is the lexicographically first power product within its equivalence class. Since every two standard monomials are mapped to different power products in $L[Y]$, the residue classes in $L[X] / \operatorname{ker} f$ of the standard monomials are linearly independent over $L$. Let $\Sigma$ be the set of 
nonstandard power products in $\mathbf{N}^{X}$. This is an ideal of monomials, for, given an element $M \in \Sigma$ and a variable $X_{a} \in X$, there is some $M^{\prime}$ in the equivalence class of $M$ such that $M^{\prime}<M$. But then $X_{a} M^{\prime} \sim X_{a} M$ and $X_{a} M^{\prime}<X_{a} M$, hence $X_{a} M \in \Sigma$. The ideal of monomials $\Sigma$ is generated by its power products of degree two. To see this, let $M \in \Sigma$. Note that the degree of $M$ is at least two. We have to show that some element $X_{a} X_{b} \in \Sigma$ divides $M$. Let $M^{\prime}$ be some power product in the equivalence class of $M$ such that $M^{\prime}<M$. In case there is some $X_{c} \in X$ dividing both $M^{\prime}$ and $M$, the quotient $M / X_{c}$ is in $\Sigma$ and it is sufficient to prove the claim for $M / X_{c}$ instead of $M$. So assume the greatest common divisor of $M^{\prime}$ and $M$ is trivial. Choose variables $X_{a}, X_{a^{\prime}} \in X$ such that $X_{a} \mid M$ and $X_{a^{\prime}} \mid M^{\prime}$, and such that they are the lexicographically first variables in $X$ with these properties. Then $X_{a^{\prime}} \leq X_{a}$ and, since the greatest common divisor of $M$ and $M^{\prime}$ is trivial, $X_{a^{\prime}}<X_{a}$.

Let $r \in \mathbf{N}$ be the minimal integer such that $a^{\prime}(r) \neq a(r)$. Then $a^{\prime}(r)>a(r)$. Since $\left|a^{\prime}\right|=m=|a|$, there is some $s>r$ with $a(s)>a^{\prime}(s) \geq 0$. Further, since $M \sim M^{\prime}$ and $a^{\prime}(r)>a(r)$, there exists some variable $X_{b}$ dividing $M / X_{a}$ such that $b(r)>0$. Let $a^{\prime \prime}:=a+e_{r}-e_{s}$ and $b^{\prime \prime}:=b-e_{r}+e_{s}$ with $e_{i}=$ $(0, \ldots, 1, \ldots, 0)$ the $i$ th coordinate vector in $(n+1)$-space $(i=0, \ldots, n)$. Then $X_{a^{\prime \prime}}$ and $X_{b^{\prime \prime}}$ are well-defined variables in $X$ such that $X_{a^{\prime \prime}}<X_{a} \leq X_{b}<$ $X_{b^{\prime \prime}}$ and $a+b=a^{\prime \prime}+b^{\prime \prime}$. In particular, $X_{a^{\prime \prime}} X_{b^{\prime \prime}}<X_{a} X_{b}$, hence $X_{a} X_{b} \in \Sigma$. By construction, $X_{a} X_{b} \mid M$. We have proved that the generators of $\Sigma$ are the power products of degree two in $\Sigma$.

Let $X_{a} X_{b} \in \Sigma$ be some generator. Take the lexicographically first power product $X_{a^{\prime}} X_{b^{\prime}}$ in the equivalence class of $X_{a} X_{b}$. Then

$$
X_{a} X_{b} \equiv X_{a^{\prime}} X_{b^{\prime}} \quad \bmod (\operatorname{ker} f) .
$$

We may assume $X_{a} \leq X_{b}$ and $X_{a^{\prime}} \leq X_{b^{\prime}}$. Note that $X_{a^{\prime}} \leq X_{a}$, since $X_{a^{\prime}} X_{b^{\prime}}<$ $X_{a} X_{b}$. In view of $a+b=a^{\prime}+b^{\prime}$ equality $X_{a^{\prime}}=X_{a}$ would imply $X_{b^{\prime}}=X_{b}$, which is impossible. Therefore,

$$
X_{a^{\prime}}<X_{a} \leq X_{b} .
$$

We have proved $\operatorname{gr}\left(B_{0}\right)=L[X] / \operatorname{ker} f$ has a Hodge algebra structure over $L$ on the poset $X$ governed by the ideal of monomials $\Sigma$ with the straightening relations given by (39) (see [DEP, Proposition 1.1]).

We want to show that $\widehat{B}_{0} \cong R_{0} / I_{0}$ with $R_{0}:=L[[X]]$ and $I_{0}:=(\operatorname{ker} f) R_{0}$ has only tangentially flat deformations. By Proposition (4.6) it is sufficient to show

$$
N_{\mathrm{gr}_{F, \alpha}\left(R_{0}, I_{0}\right)}(<-1)=0 .
$$

Here $F R_{0}$ is the filtration associated with the weight function

$$
\operatorname{ord}\left(X_{a_{1}}^{v(1)} \cdots X_{a_{k}}^{v(k)}\right):=\sum_{i=1}^{k} v(i) \cdot 3 \operatorname{dim} X_{a_{i}}
$$


(see [DEP, proof of Proposition 1.1]) and $\alpha$ is the usual semigroup structure on $\mathbf{N}, \alpha\left(d, d^{\prime}\right):=d+d^{\prime}$. Note that, since ord is additive, condition (34) of (4.6) is satisfied, so that the ring $\operatorname{gr}_{F, \alpha}\left(R_{0}\right)$ can be identified with the polynomial ring $L[X]$. The module $N_{\mathrm{gr}_{F, \alpha}\left(R_{0}, I_{0}\right)}$ is to be considered to have the graded structure coming from the total degree graded structure of $\operatorname{gr}_{F, \alpha}\left(R_{0}\right)=L[X]$.

Since every $M \in \Sigma$ can be expressed in $R_{0} / I_{0}$ as a linear combination of standard monomials of strictly greater weight,

$$
\operatorname{gr}_{F, \alpha}\left(R_{0} / I_{0}\right) \cong L[X] / \Sigma L[X]
$$

as graded algebras over $L$. Let $S$ be the set of generators of $\Sigma$. By Proposition (3.6) it is sufficient to show that every 1-component of $S$ has at least two elements, with the exception of the case $(m, n)=(2,1)$, when we must show that $B_{0}=R_{0} / I_{0}$ is a complete intersection. All this follows directly from the lemma below.

(4.13) Lemma. Let $X_{a}, X_{b} \in X$ be such that $X_{a} \leq X_{b}$. Then

(i) $X_{a} X_{b} \in S$, if and only if there are integers $r$, such that $0 \leq r<s \leq n$ and $b(r)>0, a(s)>0$.

(ii) If $X_{a} X_{b}$ is in $S$ and is the only element of its 1-component, then $(m, n)=$ $(2,1), a=b=(1,1)$, and $\operatorname{gr}_{F, \alpha}\left(R_{0} / I_{0}\right) \cong L[X] / \Sigma L[X]$ is a complete intersection.

Proof. (i) Suppose $X_{a} X_{b} \in S$. Let $X_{a^{\prime}} X_{b^{\prime}} \quad\left(<X_{a} X_{b}\right)$ be the lexicographically first power product in the equivalence class of $X_{a} X_{b}$. We may assume $X_{a^{\prime}} \leq$ $X_{b^{\prime}}$. Then $X_{a^{\prime}} \leq X_{a}$. Equality $X_{a^{\prime}}=X_{a}$ would imply $X_{b^{\prime}}=X_{b}$, since $a+b=a^{\prime}+b^{\prime}$, which is impossible. Therefore $X_{a^{\prime}}<X_{a}$, hence $X_{b^{\prime}}>X_{b}$. Together,

$$
X_{a^{\prime}}<X_{a} \leq X_{b}<X_{b^{\prime}} .
$$

Let $r \in \mathbf{N}$ be the minimum integer such that $a^{\prime}(r) \neq a(r)$. Then $a^{\prime}(r)>a(r)$. Since $\left|a^{\prime}\right|=m=|a|$, there is some $s>r$ with $a(s)>a^{\prime}(s)(\geq 0)$. Further, from $a+b=a^{\prime}+b^{\prime}$ and $a^{\prime}(r)>a(r)$ we deduce $b(r)>b^{\prime}(r)(\geq 0)$.

Conversely, suppose there are integers $r, s$ such that $0 \leq r<s \leq n$ and $b(r), a(s)>0$. Define $a^{\prime}:=a+e_{r}-e_{s}$ and $b^{\prime}:=b-e_{r}+e_{s}$. Then $X_{a^{\prime}}$ and $X_{b^{\prime}}$ are well-defined elements of $X$ satisfying $X_{a^{\prime}}<X_{a} \leq X_{b}<X_{b^{\prime}}$ and $a+b=a^{\prime}+b^{\prime}$. In particular $X_{a^{\prime}} X_{b^{\prime}} \sim X_{a} X_{b}$ and $X_{a^{\prime}} X_{b^{\prime}}<X_{a} X_{b}$, hence $X_{a} X_{b} \in S$.

(ii) Assume $X_{a} X_{b}$ is in $S$ and is the only element in its 1-component in $S$. Then there does not exist any variable $X_{c}$ different from $X_{a}$ and $X_{b}$ such that $X_{a} X_{c}$ or $X_{b} X_{c}$ is in $S$. We may assume $X_{a} \leq X_{b}$. Then, by the first part of the lemma, there are integers, $r, s$ such that $0 \leq r<s \leq n, b(r), a(s)>0$. In case $b(r)>1$, letting $c:=b+e_{s}-e_{r}$, one arrives at a contradiction, since $X_{a} \leq X_{b}<X_{c}$ and $X_{a} X_{c} \in S$ by (i). Therefore, $b(r)=1$. If $a(s)>1$, let $c:=a+e_{r}-e_{s}$. Then $X_{c}<X_{a} \leq X_{b}$ and $X_{c} X_{b} \in S$, again contradicting our assumption. So $a(s)=1$. If $a(0)<m-1$, there exists some $t \neq 0, s$ with $a(t)>0$. Define $c:=a+e_{0}-e_{t}$. Then $X_{c}<X_{a} \leq X_{b}$ and $X_{c} X_{b} \in S$. 
This shows $a(0)=m-1$. If $b(n)<m-1$, there is some $t \neq r, n$ with $b(t)>0$. Let $c:=b+e_{n}-e_{t}$. Then $X_{a} \leq X_{b}<X_{c}$ and $X_{a} X_{c} \in S$. Therefore $b(n)=m-1$. In case $n>1$ at least one coordinate of $a$ and of $b$ is zero. There is a shift in the coordinates of $a$ or $b$ giving again a variable, which is assumed not to exist. We see that

$$
n=1, \quad a=(m-1,1), \quad b=(1, m-1) .
$$

If $m \geq 4$, letting $c:=(m-2,2)$, we get a contradiction, since $X_{c}<X_{b}$ and $X_{c} X_{b} \in S$. So $m \leq 3$.

If $m=3, a=(2,1)$ and $b=(1,2)$. But then $X_{b}^{2}$ is in the 1-component of $X_{a} X_{b}$ and

$$
X_{b}^{2}=X_{1,2}^{2} \sim X_{2,1} X_{0,3}<X_{b}^{2},
$$

i.e., $X_{b}^{2} \in S$.

If $m=2, a=b=(1,1)$. Further $X=\left\{X_{2,0}, X_{1,1}, X_{0,2}\right\}$ and $S=$ $\left\{X_{1,1}^{2}\right\}$. Therefore, $\operatorname{gr}_{F, \alpha}\left(B_{0}\right) \cong L[H] / \Sigma L[H]$ is a complete intersection, and hence so is $B_{0}$.

If $m=1, a=(0,1)$ and $b=(1,0)$, contradicting the assumption that $X_{a} \leq X_{b}$. Q.E.D.

\section{REFERENCES}

[Bo] N. Bourbaki, Algèbre commutative, Hermann, Paris, 1961-1965.

[DEP] C. DeConcini, D. Eisenbud, and C. Procesi, Hodge algebras, Asterisque Soc. Math. France 91 (1982).

[GD] A. Grothendieck and J. Dieudonné, Eléments de géométrie algébrique, Inst. Hautes Études Sci. Publ. Math., Paris, 1960-1967.

[Ha] R. Hartshorne, Algebraic geometry, Springer, Berlin, 1977.

$\left[\mathrm{He}_{1}\right]$ B. Herzog, Die Wirkung lokaler Homomorphismen auf die Hilbertfunktion, Math. Nachr. 97 (1980), 103-115.

$\left[\mathrm{He}_{2}\right]+$ On a relation between the Hilbert functions belonging to a local homomorphism, $\mathrm{J}$. London Math. Soc. 25 (1982), 458-466.

$\left[\mathrm{He}_{3}\right]$ - A criterion for tangential flatness, Manuscripta Math. 43 (1983), 219-228.

[He] J. Herzog, Strict local rings, Proc. Amer. Math. Soc. 84 (1982), 165-172.

[Hi] H. Hironaka, Certain numerical characters of singularities, J. Math. Kyoto Univ. 10 (1970), 151-187.

[I] A. Iarrobino, Compressed algebras, Trans. Amer. Math. Soc. 285 (1984), 337-378.

[L] C. Lech, Inequalities related to certain couples of local rings, Acta Math. 112 (1964), 69-89.

[Ma] H. Matsumura, Commutative ring theory, Cambridge Univ. Press, 1986.

[Sche] P. Schenzel, Über die freien Auflösungen extremaler Cohen-Macaulay Ringe, J. Algebra 64 (1980), 93-101. 